\title{
STRONG CONVERGENCE TO THE HOMOGENIZED LIMIT OF ELLIPTIC EQUATIONS WITH RANDOM COEFFICIENTS
}

\author{
JOSEPH G. CONLON AND THOMAS SPENCER
}

\begin{abstract}
Consider a discrete uniformly elliptic divergence form equation on the $d$ dimensional lattice $\mathbf{Z}^{d}$ with random coefficients. It has previously been shown that if the random environment is translational invariant, then the averaged Green's function, together with its first and second differences, are bounded by the corresponding quantities for the constant coefficient discrete elliptic equation. It has also been shown that if the random environment is ergodic, then solutions of the random equation converge under diffusive scaling to solutions of a homogenized elliptic PDE on $\mathbf{R}^{d}$. In this paper point-wise estimates are obtained on the difference between the averaged Green's function and the homogenized Green's function for certain random environments which are strongly mixing.
\end{abstract}

\section{INTRODUCTION}

Let $(\Omega, \mathcal{F}, P)$ be a probability space and denote by $\langle\cdot\rangle$ expectation w.r. to the measure $P$. We assume that the $d$ dimensional integer lattice $\mathbf{Z}^{d}$ acts on $\Omega$ by translation operators $\tau_{x}: \Omega \rightarrow \Omega, x \in \mathbf{Z}^{d}$, which are measure preserving and satisfy the properties $\tau_{x} \tau_{y}=\tau_{x+y}, \tau_{0}=$ identity, $x, y \in \mathbf{Z}^{d}$. Consider a bounded measurable function $\mathbf{a}: \Omega \rightarrow \mathbf{R}^{d(d+1) / 2}$ from $\Omega$ to the space of symmetric $d \times d$ matrices which satisfies the quadratic form inequality

$$
\lambda I_{d} \leq \mathbf{a}(\omega) \leq \Lambda I_{d}, \quad \omega \in \Omega,
$$

where $I_{d}$ is the identity matrix in $d$ dimensions and $\Lambda, \lambda$ are positive constants. We shall be interested in solutions $u(x, \eta, \omega)$ to the discrete elliptic equation

$$
\eta u(x, \eta, \omega)+\nabla^{*} \mathbf{a}\left(\tau_{x} \omega\right) \nabla u(x, \eta, \omega)=h(x), \quad x \in \mathbf{Z}^{d}, \omega \in \Omega .
$$

In (1.2) we take $\eta \geq 0$ and $\nabla$ the discrete gradient operator, which has adjoint $\nabla^{*}$. Thus $\nabla$ is a $d$ dimensional column operator and $\nabla^{*}$ a $d$ dimensional row operator, which act on functions $\phi: \mathbf{Z}^{d} \rightarrow \mathbf{R}$ by

$$
\begin{array}{rll}
\nabla \phi(x) & =\left(\nabla_{1} \phi(x), \ldots, \quad \nabla_{d} \phi(x)\right), & \nabla_{i} \phi(x)=\phi\left(x+\mathbf{e}_{i}\right)-\phi(x), \\
\nabla^{*} \phi(x) & =\left(\nabla_{1}^{*} \phi(x), \ldots, \nabla_{d}^{*} \phi(x)\right), & \nabla_{i}^{*} \phi(x)=\phi\left(x-\mathbf{e}_{i}\right)-\phi(x) .
\end{array}
$$

In (1.3) the vector $\mathbf{e}_{i} \in \mathbf{Z}^{d}$ has 1 as the $i$ th coordinate and 0 for the other coordinates, $1 \leq i \leq d$.

It is well known [14,20, 25] that if the translation operators $\tau_{x}, x \in \mathbf{Z}^{d}$, are ergodic on $\Omega$, then solutions to the random equation (1.2) converge to solutions

Received by the editors March 1, 2011 and, in revised form, November 23, 2011.

2010 Mathematics Subject Classification. Primary 81T08, 82B20, 35R60, 60J75.

Key words and phrases. Euclidean field theory, PDE with random coefficients, homogenization. 
of a constant coefficient equation under suitable scaling. Thus suppose $f: \mathbf{R}^{d} \rightarrow$ $\mathbf{R}$ is a $C^{\infty}$ function with compact support, and for $\varepsilon$ satisfying $0<\varepsilon \leq 1$ set $h(x)=\varepsilon^{2} f(\varepsilon x), x \in \mathbf{Z}^{d}$, in (1.2). Then $u\left(x / \varepsilon, \varepsilon^{2} \eta, \omega\right)$ converges with probability 1 as $\varepsilon \rightarrow 0$ to a function $u(x, \eta), x \in \mathbf{R}^{d}$, which is the solution to the constant coefficient elliptic PDE

$$
\eta u(x, \eta)+\nabla^{*} \mathbf{a}_{\mathrm{hom}} \nabla u(x, \eta)=f(x), \quad x \in \mathbf{R}^{d},
$$

where the $d \times d$ symmetric matrix $\mathbf{a}_{\text {hom }}$ satisfies the quadratic form inequality (1.1). This homogenization result can be viewed as a kind of central limit theorem, and our purpose here will be to show that the theorem can be strengthened for certain probability spaces $(\Omega, \mathcal{F}, P)$.

We consider what the homogenization result says about the expectation of the Green's function for equation (1.2). By translation invariance of the measure we have that

$$
\langle u(x, \eta, \cdot)\rangle=\sum_{y \in \mathbf{Z}^{d}} G_{\mathbf{a}, \eta}(x-y) h(y), \quad x \in \mathbf{Z}^{d},
$$

where $G_{\mathbf{a}, \eta}(x)$ is the expected value of the Green's function. Setting $h(x)=$ $\varepsilon^{2} f(\varepsilon x), x \in \mathbf{Z}^{d}$, then (1.5) may be written as

$$
\left\langle u\left(x / \varepsilon, \varepsilon^{2} \eta, \cdot\right)\right\rangle=\int_{\varepsilon Z^{d}} \varepsilon^{2-d} G_{\mathbf{a}, \varepsilon^{2} \eta}\left(\frac{x-z}{\varepsilon}\right) f(z) d z, \quad x \in \varepsilon \mathbf{Z}^{d},
$$

where integration over $\varepsilon \mathbf{Z}^{d}$ is defined by

$$
\int_{\varepsilon Z^{d}} g(z) d z=\sum_{z \in \varepsilon \mathbf{Z}^{d}} g(z) \varepsilon^{d} .
$$

Let $G_{\mathbf{a}_{\mathrm{hom}, \eta}}(x), x \in \mathbf{R}^{d}$, be the Green's function for the PDE (1.4). One easily sees that $G_{\mathbf{a}_{\text {hom }}, \eta}(\cdot)$ satisfies the scaling property

$$
\varepsilon^{2-d} G_{\mathbf{a}_{\mathrm{hom},}, \varepsilon^{2} \eta}(x / \varepsilon)=G_{\mathbf{a}_{\mathrm{hom}, \eta}}(x), \quad \varepsilon, \eta>0, x \in \mathbf{R}^{d}-\{0\} .
$$

From (1.6) and (1.8) we see that homogenization implies that the function $\varepsilon^{2-d} G_{\mathbf{a}, \varepsilon^{2} \eta}(x / \varepsilon), x \in \varepsilon \mathbf{Z}^{d}$, converges in an averaged sense to the Green's function $G_{\mathbf{a}_{\text {hom }}, \eta}(x), x \in \mathbf{R}^{d}$. A consequence of our results here will be that for certain probability spaces $(\Omega, \mathcal{F}, P)$ and functions $\mathbf{a}: \Omega \rightarrow \mathbf{R}^{d(d+1) / 2}$ this convergence is point-wise in $x$. In particular, for some $\alpha$ satisfying $0<\alpha \leq 1$, there are positive constants $C, \gamma$ such that

$$
\begin{aligned}
&\left|\varepsilon^{2-d} G_{\mathbf{a}, \varepsilon^{2} \eta}(x / \varepsilon)-G_{\mathbf{a}_{\mathrm{hom}}, \eta}(x)\right| \leq \frac{C \varepsilon^{\alpha}}{[|x|+\varepsilon]^{d-2+\alpha}} e^{-\gamma \sqrt{\eta / \Lambda}|x|} \\
& 0<\varepsilon \leq 1, x \in \varepsilon \mathbf{Z}^{d}-\{0\} .
\end{aligned}
$$

It is clear that the inequality (1.9) for $\varepsilon<1$ follows from the same inequality for $\varepsilon=1$, i.e.

$$
\left|G_{\mathbf{a}, \eta}(x)-G_{\mathbf{a}_{\mathrm{hom}}, \eta}(x)\right| \leq \frac{C}{\Lambda(|x|+1)^{d-2+\alpha}} e^{-\gamma \sqrt{\eta / \Lambda}|x|}, \quad x \in \mathbf{Z}^{d}-\{0\},
$$

provided we are able to obtain an inequality (1.10) which is uniform in $\eta>0$ as $\eta \rightarrow 0$. We shall prove such an inequality and also similar inequalities for the 
derivatives of the expectation of the Green's function,

$$
\begin{aligned}
& \left|\nabla G_{\mathbf{a}, \eta}(x)-\nabla G_{\mathbf{a}_{\mathrm{hom}}, \eta}(x)\right| \leq \frac{C}{\Lambda(|x|+1)^{d-1+\alpha}} e^{-\gamma \sqrt{\eta / \Lambda}|x|}, \quad x \in \mathbf{Z}^{d}-\{0\}, \\
& \left|\nabla \nabla G_{\mathbf{a}, \eta}(x)-\nabla \nabla G_{\mathbf{a}_{\mathrm{hom}}, \eta}(x)\right| \leq \frac{C}{\Lambda(|x|+1)^{d+\alpha}} e^{-\gamma \sqrt{\eta / \Lambda}|x|} \quad x \in \mathbf{Z}^{d}-\{0\} .
\end{aligned}
$$

Theorem 1.1. Suppose $\mathbf{a}(\cdot)$ satisfies (1.1), the matrices $\mathbf{a}\left(\tau_{x} \cdot\right), x \in \mathbf{Z}^{d}$, are independent, and $0<\eta \leq \Lambda$. Then for $d \geq 2$ there exists $\alpha>0$ depending only on $d$ and $\Lambda / \lambda$, such that (1.10), (1.11) and (1.12) hold for some positive constants $\gamma, C$, depending only on $d$ and $\Lambda / \lambda$.

We also consider here probability spaces $(\Omega, \mathcal{F}, P)$ corresponding to certain Euclidean field theories. These Euclidean field theories are determined by a potential $V: \mathbf{R}^{d} \rightarrow \mathbf{R}$ which is a $C^{2}$ uniformly convex function. Thus the second derivative $\mathbf{a}(\cdot)=V^{\prime \prime}(\cdot)$ of $V(\cdot)$ is assumed to satisfy the inequality (1.1). Next consider functions $\phi: \mathbf{Z}^{d} \rightarrow \mathbf{R}$ on the integer lattice in $\mathbf{R}^{d}$. Let $\Omega$ be the space of all such functions and $\mathcal{F}$ be the Borel algebra generated by finite dimensional rectangles $\left\{\phi \in \Omega:\left|\phi\left(x_{i}\right)-a_{i}\right|<r_{i}, i=1, \ldots, N\right\}, x_{i} \in \mathbf{Z}^{d}, a_{i} \in \mathbf{R}, r_{i}>0, i=$ $1, \ldots, N, N \geq 1$. The translation operators $\tau_{x}: \Omega \rightarrow \Omega, x \in \mathbf{Z}^{d}$, are defined by $\tau_{x} \phi(z)=\phi(x+z), z \in \mathbf{Z}^{d}$. For any $d \geq 1$ and $m>0$ one can define [4,9] a unique ergodic translation invariant probability measure $P$ on $(\Omega, \mathcal{F})$ which depends on the function $V$ and $m$. The measure is formally given as

$$
\exp \left[-\sum_{x \in \mathbf{Z}^{d}} V(\nabla \phi(x))+m^{2} \phi(x)^{2}\right] \prod_{x \in \mathbf{Z}^{d}} d \phi(x) / \text { normalization. }
$$

Theorem 1.2. Let $\tilde{\mathbf{a}}: \mathbf{R} \rightarrow \mathbf{R}^{d(d+1) / 2}$ be a $C^{1}$ function on $\mathbf{R}$ with values in the space of symmetric $d \times d$ matrices which satisfy the quadratic form inequality (1.1). Let $(\Omega, \mathcal{F}, P)$ be the probability space of fields $\phi(\cdot)$ determined by (1.13), and set $\mathbf{a}(\cdot)$ in (1.2) to be $\mathbf{a}(\phi)=\tilde{\mathbf{a}}(\phi(0)), \phi \in \Omega$. Suppose in addition that the derivative D $\tilde{\mathbf{a}}(\cdot)$ of $\tilde{\mathbf{a}}(\cdot)$ satisfies the inequality $\|D \tilde{\mathbf{a}}(\cdot)\|_{\infty} \leq \Lambda_{1}$. Then for $d \geq 2$ there exists $\alpha>0$ depending only on $d$ and $\Lambda / \lambda$, such that (1.10), (1.11) and (1.12) hold for some positive constants $\gamma$ and $C=C_{1}\left[\Lambda_{1} / m \Lambda+1\right]$, where $\gamma, C_{1}$ depend only on $d$ and $\Lambda / \lambda$.

The limit as $m \rightarrow 0$ of the measure (1.13) is a probability measure on gradient fields $\omega: \mathbf{Z}^{d} \rightarrow \mathbf{R}^{d}$, where formally $\omega(x)=\nabla(\phi(x)), x \in \mathbf{Z}^{d}$. This massless field theory measure is ergodic with respect to translation operators [4, 9] for all $d \geq 1$. In the case $d=1$ it has a simple structure since then the variables $\omega(x), x \in \mathbf{Z}$, are i.i.d. Note that in the probability space $(\Omega, \mathcal{F}, P)$ for the massless field theory, the Borel algebra $\mathcal{F}$ is generated by the intersection of finite dimensional rectangles and the hyperplanes imposing the gradient constraints for $\omega(\cdot)$. For $d \geq 3$ the gradient field theory measure induces a measure on fields $\phi: \mathbf{Z}^{d} \rightarrow \mathbf{R}$ which is simply the limit of the measures (1.13) as $m \rightarrow 0$. For $d=1,2$ the $m \rightarrow 0$ limit of the measures (1.13) on fields $\phi: \mathbf{Z}^{d} \rightarrow \mathbf{R}$ does not exist.

We can show that the inequalities (1.10), (1.11), (1.12) also hold when $(\Omega, \mathcal{F}, P)$ is given by the massless field theory environment.

Theorem 1.3. Let $\tilde{\mathbf{a}}: \mathbf{R}^{d} \rightarrow \mathbf{R}^{d(d+1) / 2}$ be a $C^{1}$ function on $\mathbf{R}^{d}$ with values in the space of symmetric $d \times d$ matrices which satisfy the quadratic form inequality (1.1). 
Let $(\Omega, \mathcal{F}, P)$ be the probability space of gradient fields $\omega(\cdot)=\nabla \phi(\cdot)$ determined by the limit of (1.13) as $m \rightarrow 0$, and set $\mathbf{a}(\cdot)$ in (1.2) to be $\mathbf{a}(\omega)=\tilde{\mathbf{a}}(\omega(0))$, $\omega \in$ $\Omega$. Suppose in addition that the derivative D $\tilde{\mathbf{a}}(\cdot)$ of $\tilde{\mathbf{a}}(\cdot)$ satisfies the inequality $\|D \tilde{\mathbf{a}}(\cdot)\|_{\infty} \leq \Lambda_{1}$. Then for $d \geq 2$ there exists $\alpha>0$ depending only on $d$ and $\Lambda / \lambda$, such that (1.10), (1.11) and (1.12) hold for some positive constants $\gamma$ and $C=C_{1}\left[\Lambda_{1} / \Lambda \sqrt{\lambda}+1\right]$, where $\gamma, C_{1}$ depend only on $d$ and $\Lambda / \lambda$.

Our method of proof for Theorems 1.1-1.3 combine methods used to prove regularity of averaged Green's functions for PDE with random coefficients with methods for obtaining rates of convergence in homogenization. Regularity of averaged Green's functions was first proved in [6]. The results of that paper imply that for any probability space $(\Omega, \mathcal{F}, P)$ with translation invariant operators $\tau_{x}: \Omega \rightarrow \Omega, x \in \mathbf{Z}^{d}$, the inequalities (1.10), (1.11) hold for $\alpha=0$ and (1.12) for any $\alpha<0$. The approach of the paper is to obtain good control on the Fourier transform $\hat{G}_{\mathbf{a}, \eta}(\xi), \xi \in[-\pi, \pi]^{d}$, of $G_{\mathbf{a}, \eta}(x), x \in \mathbf{Z}^{d}$, for $|\xi|$ close to 0 . Using the Fourier inversion formula, one then obtains the inequalities (1.10)-(1.12). In [7] the inequality (1.12) is proven with $\alpha=0$, and in fact Hölder continuity of the second difference of $G_{\mathbf{a}, \eta}(x), x \in \mathbf{Z}^{d}$, is also established. In contrast to [6], the approach of [7] is local in configuration space, and uses results from harmonic analysis which are deeper than those used in [6]. In particular, the Harnack inequality [12 for uniformly elliptic equations in divergence form is needed to prove (1.12) with $\alpha=0$, whereas the proof of (1.12) with $\alpha<0$ in [6] follows from interpolation inequalities.

We have already observed that the inequality (1.10) with $\alpha>0$ implies (1.9), which gives a rate of convergence of $\varepsilon^{\alpha}$ in homogenization. In $\S 8$ we show how the methodology used in proving Theorems 1.1-1.3 can also be used to obtain rate of convergence results. In particular we prove the following:

Theorem 1.4. Suppose $d \geq 2, \eta>0$, and $f: \mathbf{R}^{d} \rightarrow \mathbf{R}$ is a $C^{\infty}$ function of compact support. Let $u_{\varepsilon}(x, \eta, \omega)$ be the solution to (1.2) with $h(x)=\varepsilon^{2} f(\varepsilon x), x \in \mathbf{Z}^{d}$, and $u_{\mathrm{hom}}(x, \eta), x \in \mathbf{R}^{d}$, the solution to (1.4). We further assume that $(\Omega, \mathcal{F}, P)$ is either the independent variable environment of Theorem 1.1, the massive field theory environment of Theorem 1.2 or the massless field theory environment of Theorem 1.3. Then there is a constant $\alpha>0$ depending only on $d, \Lambda / \lambda$ and $a$ constant $C$ independent of $\varepsilon$ such that

$$
\sup _{x \in \varepsilon \mathbf{Z}^{d}}\left\langle\left|u_{\varepsilon}\left(x / \varepsilon, \varepsilon^{2} \eta, \cdot\right)-u_{\text {hom }}(x, \eta)\right|^{2}\right\rangle \leq C \varepsilon^{\alpha}, \quad \text { for } 0<\varepsilon \leq 1 .
$$

The first results establishing a rate of convergence for homogenization of elliptic PDE in divergence form were obtained by Yurinskii in [24]. Yurinskii's result is stronger than (1.14) since he proves that

$$
\left\langle\sup _{x \in \varepsilon \mathbf{Z}^{d}}\left|u_{\varepsilon}\left(x / \varepsilon, \varepsilon^{2} \eta, \cdot\right)-u_{\text {hom }}(x, \eta)\right|^{2}\right\rangle \leq C \varepsilon^{\alpha}, \quad \text { for } 0<\varepsilon \leq 1,
$$

but it does not apply to all the environments covered by Theorem 1.4. Yurinskii's assumption on the environment is a quantitative strong mixing condition. Thus let $\chi(\cdot)$ be a positive decreasing function on $\mathbf{R}^{+}$such that $\lim _{q \rightarrow \infty} \chi(q)=0$. The quantitative strong mixing condition is given in terms of the function $\chi(\cdot)$ as follows: For any subsets $A, B$ of $\mathbf{Z}^{d}$ and events $\Gamma_{A}, \Gamma_{B} \subset \Omega$, which depend respectively only on variables $\phi(x), x \in A$, and $\phi(y), y \in B$, then

$$
\left|P\left(\Gamma_{A} \cap \Gamma_{B}\right)-P\left(\Gamma_{A}\right) P\left(\Gamma_{B}\right)\right| \leq \chi\left(\inf _{x \in A, y \in B}|x-y|\right) .
$$


In the proof of (1.15) he requires the function $\chi(\cdot)$ to have power law decay, i.e. $\lim _{q \rightarrow \infty} q^{\alpha} \chi(q)=0$ for some $\alpha>0$. This condition evidently holds for the independent variable environment of Theorem 1.1, but is only known to hold for the massive and massless field theory environments in the Gaussian case where the function $V(\cdot)$ of (1.13) is quadratic. Recently Caffarelli and Souganidis [3] have obtained rates of convergence results in homogenization of fully nonlinear PDE under the quantitative strong mixing condition (1.16). In their case the function $\chi(q)$ is assumed to decay logarithmically in $q$ to 0 , and correspondingly the rate of convergence in homogenization that is obtained is also logarithmic in $\varepsilon$. In the fully nonlinear case a stronger assumption on the function $\chi(\cdot)$, for example power law decay, does not yield a stronger rate of convergence in homogenization.

Rates of convergence in homogenization when $(\Omega, \mathcal{F}, P)$ is either the massive field theory of Theorem 1.2 or the massless field theory of Theorem 1.3 were first obtained in 18. The main tools used to prove these results are the Brascamp-Lieb (BL) inequality [1] and Meyer's theorem [15]. Meyer's theorem is a consequence of the continuity in $p$ of the norms of Calderon-Zygmund operators acting on the spaces $L^{p}\left(\mathbf{Z}^{d}\right)$ of functions whose $p$ th powers are summable. In [5] it was shown that Meyer's theorem could also be used to obtain a rate of convergence in the independent variable case of Theorem 1.1. In recent work of Gloria and Otto [10,11] the independent variable case was taken up again, showing that the method of [18] can also be implemented in this case by assuming a weakened version of the BL inequality. Because of the perturbative nature of Meyer's theorem, the method of [18] alone does not yield optimal rates of convergence in homogenization. However, by combining the method with some deterministic estimates on Green's functions, optimal rates of convergence to homogenization are obtained in [10, 11].

In the present paper we follow the methodology of [6] to obtain estimates on $\hat{G}_{\mathbf{a}, \eta}(\xi), \xi \in[-\pi, \pi]^{d}$, which imply (1.10), 1.11) with $\alpha=0$. The estimates on $\hat{G}_{\mathbf{a}, \eta}(\xi)$ are improved by the use of Meyer's theorem for the independent variable environment, and by the BL inequality plus Meyer's theorem in the field theory case. The inequalities (1.10), (1.11) for some $\alpha>0$ then follow upon using the Fourier inversion formula. To prove (1.12) we also have to use the results of [7] to estimate the contribution of high Fourier modes. These estimates are a consequence of the Hölder continuity of the second difference of $G_{\mathbf{a}, \eta}(x), x \in \mathbf{Z}^{d}$, already mentioned.

\section{Fourier space RePRESEntation AND estimates}

In this section we summarize relevant results of previous work 5,6 which were used to prove pointwise bounds on the Green's function $G_{\mathbf{a}, \eta}(x), x \in \mathbf{Z}^{d}$, defined by (1.5). The starting point for this is the Fourier representation

$$
G_{\mathbf{a}, \eta}(x)=\frac{1}{(2 \pi)^{d}} \int_{[-\pi, \pi]^{d}} \frac{e^{-i \xi \cdot x}}{\eta+e(\xi)^{*} q(\xi, \eta) e(\xi)} d \xi,
$$

where the $d \times d$ matrix function $q(\xi, \eta), \xi \in \mathbf{R}^{d}, \eta>0$, is a complex Hermitian positive definite function of $(\xi, \eta)$, periodic in $\xi$ with fundamental region $[-\pi, \pi]^{d}$, which satisfies the quadratic form inequality

$$
\lambda I_{d} \leq q(\xi, \eta) \leq \Lambda I_{d}, \quad \xi \in \mathbf{R}^{d}, \eta>0 .
$$

The $d$ dimensional column vector $e(\xi)$ in (2.1) has $j$ th entry $e_{j}(\xi)=e^{-i \mathbf{e}_{j} \cdot \xi}-1,1 \leq$ $j \leq d$. 
The function $q(\cdot, \cdot)$ is given in terms of the solution of an elliptic equation on $\Omega$. For a measurable function $\psi: \Omega \rightarrow \mathbf{C}$ we define the $\xi$ derivative of $\psi(\cdot)$ in the $j$ direction $\partial_{j, \xi}$, and its adjoint $\partial_{j, \xi}^{*}$, by

$$
\begin{aligned}
& \partial_{j, \xi} \psi(\omega)=e^{-i \mathbf{e}_{j} \cdot \xi} \psi\left(\tau_{\mathbf{e}_{j}} \omega\right)-\psi(\omega), \\
& \partial_{j, \xi}^{*} \psi(\omega)=e^{i \mathbf{e}_{j} \cdot \xi} \psi\left(\tau_{-\mathbf{e}_{j}} \omega\right)-\psi(\omega) .
\end{aligned}
$$

We also define a $d$ dimensional column $\xi$ derivative operator $\partial_{\xi}$ by $\partial_{\xi}=\left(\partial_{1, \xi}, \ldots, \partial_{d, \xi}\right)$, which has adjoint $\partial_{\xi}^{*}$ given by the row operator $\partial_{\xi}^{*}=\left(\partial_{1, \xi}^{*}, \ldots, \partial_{d, \xi}^{*}\right)$. Let $\Phi(\xi, \eta, \omega)$ be the $d$ dimensional row vector which is the solution to the equation

$$
\eta \Phi(\xi, \eta, \omega)+P \partial_{\xi}^{*} \mathbf{a}(\omega) \partial_{\xi} \Phi(\xi, \eta, \omega)=-P \partial_{\xi}^{*} \mathbf{a}(\omega), \quad \eta>0, \xi \in \mathbf{R}^{d}, \omega \in \Omega,
$$

where $P$ is the projection orthogonal to the constant function. Then $q(\xi, \eta)$ is given in terms of the solution to (2.4) by the formula

$$
q(\xi, \eta)=\langle\mathbf{a}(\cdot)\rangle+\left\langle\mathbf{a}(\cdot) \partial_{\xi} \Phi(\xi, \eta, \cdot)\right\rangle .
$$

The solution to (2.4) can be generated by a convergent perturbation expansion. Let $\mathcal{H}(\Omega)$ be the Hilbert space of measurable functions $\psi: \Omega \rightarrow \mathbf{C}^{d}$ with norm $\|\psi\|$ given by $\|\psi\|^{2}=\left\langle|\psi(\cdot)|^{2}\right\rangle$. We define an operator $T_{\xi, \eta}$ on $\mathcal{H}(\Omega)$ as follows: For any $g \in \mathcal{H}$, let $\psi(\xi, \eta, \omega)$ be the solution to the equation

$$
\frac{\eta}{\Lambda} \psi(\xi, \eta, \omega)+\partial_{\xi}^{*} \partial_{\xi} \psi(\xi, \eta, \omega)=\partial_{\xi}^{*} g(\omega), \quad \eta>0, \xi \in \mathbf{R}^{d}, \omega \in \Omega .
$$

Then $T_{\xi, \eta} g(\cdot)=\partial_{\xi} \psi(\xi, \eta, \cdot)$, or more explicitly

$$
T_{\xi, \eta} g(\omega)=\sum_{x \in \mathbf{Z}^{d}}\left\{\nabla \nabla^{*} G_{\eta / \Lambda}(x)\right\}^{*} \exp [-i x . \xi] g\left(\tau_{x} \omega\right),
$$

where $G_{\eta}(\cdot)$ is the solution to the equation

$$
\eta G_{\eta}(x)+\nabla^{*} \nabla G_{\eta}(x)=\delta(x), \quad x \in \mathbf{Z}^{d} .
$$

It is easy to see from (2.6) that $T_{\xi, \eta}$ is a bounded self-adjoint operator on $\mathcal{H}(\Omega)$ with $\left\|T_{\xi, \eta}\right\| \leq 1$, provided $\xi \in \mathbf{R}^{d}, \eta>0$. Now on setting $\mathbf{a}(\cdot)=\Lambda\left[I_{d}-\mathbf{b}(\cdot)\right]$, one sees that (2.4) is equivalent to the equation

$$
\partial_{\xi} \Phi(\xi, \eta, \cdot)=P T_{\xi, \eta}\left[\mathbf{b}(\cdot) \partial_{\xi} \Phi(\xi, \eta, \cdot)\right]+P T_{\xi, \eta}[\mathbf{b}(\cdot)] .
$$

Since $\left\|T_{\xi, \eta}\right\| \leq 1$ and $\|\mathbf{b}(\omega)\| \leq 1-\lambda / \Lambda, \omega \in \Omega$, the Neumann series for the solution to (2.9) converges in $\mathcal{H}(\Omega)$.

It will be useful later on to express the operator $T_{\xi, \eta}$ in its Fourier representation. To do this we use the standard notation for the Fourier transform $\hat{h}(\zeta), \zeta \in[-\pi, \pi]^{d}$, of a function $h: \mathbf{Z}^{d} \rightarrow \mathbf{C}$. Thus

$$
\hat{h}(\zeta)=\sum_{x \in \mathbf{Z}^{d}} h(x) e^{i x \cdot \zeta}, \quad \zeta \in \mathbf{R}^{d},
$$

and the Fourier inversion formula yields

$$
h(x)=\frac{1}{(2 \pi)^{d}} \int_{[-\pi, \pi]^{d}} \hat{h}(\zeta) e^{-i x \cdot \zeta} d \zeta, \quad x \in \mathbf{Z}^{d} .
$$

Now the action of the translation group $\tau_{x}, x \in \mathbf{Z}^{d}$, on $\Omega$ can be described by a set $A_{1}, \ldots, A_{d}$ of commuting self-adjoint operators on $L^{2}(\Omega)$, so that

$$
f\left(\tau_{x} \cdot\right)=\exp [i x \cdot A] f(\cdot), \quad x \in \mathbf{Z}^{d}, f \in L^{2}(\Omega),
$$


where $A=\left(A_{1}, \ldots, A_{d}\right)$. It then follows from (2.7) and (2.12) that

$$
T_{\xi, \eta} g(\cdot)=\frac{e(\xi-A) e^{*}(\xi-A)}{\eta / \Lambda+e(\xi-A)^{*} e(\xi-A)} g(\cdot) .
$$

It is easy to see that the function $q(\xi, \eta)$ is $C^{\infty}$ for $\xi \in \mathbf{R}^{d}, \eta>0$. In [5, 6] it was further shown that if the translation operators $\tau_{x}, x \in \mathbf{Z}^{d}$, are ergodic on $(\Omega, \mathcal{F}, P)$, then $\lim _{(\xi, \eta) \rightarrow(0,0)} q(\xi, \eta)=q(0,0)$ exists. We shall be able to extend this result by recalling the notion of weak mixing 21] of a measure preserving transformation $\tau: \Omega \rightarrow \Omega$. Like ergodicity it is a spectral property. Thus $\tau$ is weak mixing if the operator $U: L^{2}(\Omega) \rightarrow L^{2}(\Omega)$ induced by $\tau$, i.e. $U f(\cdot)=f(\tau \cdot)$ for $f \in L^{2}(\Omega)$, has exactly one eigenfunction in $L^{2}(\Omega)$, which then must be the constant function. Just as with ergodicity, weak mixing can also be characterized directly in terms of the probability measure $P$ on $\Omega$. The transformation $\tau: \Omega \rightarrow \Omega$ is weak mixing if

$$
\lim _{N \rightarrow \infty} \frac{1}{N} \sum_{n=0}^{N-1}\left|P\left(\tau^{-n} A \cap B\right)-P(A) P(B)\right|=0, \quad \text { for } A, B \in \mathcal{F} .
$$

In subsequent sections we shall establish weak mixing of transformations $\tau$ by actually proving that they are strong mixing, that is,

$$
\lim _{n \rightarrow \infty} P\left(\tau^{-n} A \cap B\right)=P(A) P(B), \quad \text { for } A, B 10 n \mathcal{F} .
$$

Thus our proof of weak mixing uses the criterion (2.14) implied by (2.15), whereas in the following proposition it is the spectral definition of weak mixing which is useful for us.

Proposition 2.1. Suppose that the operator $\tau_{\mathbf{e}_{j}}$ is weak mixing on $\Omega$ for some $j, 1 \leq j \leq d$. Then $q(\xi, \eta), \xi \in \mathbf{R}^{d}, \eta>0$, extends to a continuous function on $\xi \in \mathbf{R}^{d}, \eta \geq 0$.

Proof. We first define an operator $T_{\xi, \eta}$ on $\mathcal{H}(\Omega)$ for $\xi \in \mathbf{R}^{d}$ and $\eta=0$. To do this first observe from (2.6) that if the function $g(\cdot) \in \mathcal{H}(\Omega)$ satisfies $\partial_{\xi}^{*} g(\cdot)=0$, we should set $T_{\xi, 0} g(\cdot)=0$. Alternatively, if $g(\cdot)=\partial_{\xi} h(\cdot)$ for some $h \in L^{2}(\Omega)$, then from (2.7) we should set $T_{\xi, 0} g(\cdot)$ to be given by the formula

$$
T_{\xi, 0} g(\cdot)=\sum_{x \in \mathbf{Z}^{d}} \lim _{\eta \rightarrow 0}\left\{\nabla^{*}\left(\nabla^{*} \nabla\right) G_{\eta / \Lambda}(x)\right\}^{*} \exp [-i x . \xi] h\left(\tau_{x} \cdot\right) .
$$

In view of the inequality

$$
\left|\nabla^{*}\left(\nabla^{*} \nabla\right) G_{\eta}(x)\right| \leq C /[1+|x|]^{d+1} \quad x \in \mathbf{Z}^{d}, \eta>0,
$$

for a constant $C$ depending only on $d$, we see that the right hand side of (2.16) is in $\mathcal{H}(\Omega)$. Since the orthogonal complement in $\mathcal{H}(\Omega)$ of the null space of the operator $\partial_{\xi}^{*}$ is the closure of the linear space $\mathcal{E}_{\xi}(\Omega)=\left\{\partial_{\xi} h(\cdot): h \in L^{2}(\Omega)\right\}$, we have defined $T_{\xi, 0} g(\cdot)$ for a dense set of functions $g \in \mathcal{H}(\Omega)$ and

$$
\lim _{\eta \rightarrow 0}\left\|T_{\xi, \eta} g-T_{\xi, 0} g\right\|=0 .
$$

Using the fact that $\left\|T_{\xi, \eta}\right\| \leq 1$ for all $\eta>0$, one sees that the operator $T_{\xi, 0}$, defined above on a dense linear subspace of $\mathcal{H}(\Omega)$, extends to a bounded operator on $\mathcal{H}(\Omega)$ with norm $\left\|T_{\xi, 0}\right\| \leq 1$, and the limit (2.18) holds for all $g \in \mathcal{H}(\Omega)$. 
Having extended the operators $T_{\xi, \eta}, \xi \in \mathbf{R}^{d}, \eta>0$, to the region $\xi \in \mathbf{R}^{d}, \eta \geq 0$, we can use this fact to similarly extend the function $q(\xi, \eta)$. Thus for $m=1,2, \ldots$, let the matrix function $h_{m}(\xi, \eta)$ be defined for $\eta>0, \xi \in \mathbf{R}^{d}$ by

$$
h_{m}(\xi, \eta)=\left\langle\mathbf{b}(\cdot)\left[P T_{\xi, \eta} \mathbf{b}(\cdot)\right]^{m}\right\rangle,
$$

whence (2.5), (2.9) imply that

$$
q(\xi, \eta)=\langle\mathbf{a}(\cdot)\rangle-\Lambda \sum_{m=1}^{\infty} h_{m}(\xi, \eta)
$$

Evidently we can extend the definition of $q(\xi, \eta)$ to $\eta=0$ by setting $\eta=0$ in (2.19), (2.20). In view of (2.18) one has that $\lim _{\eta \rightarrow 0} q(\xi, \eta)=q(\xi, 0), \xi \in \mathbf{R}^{d}$, whence the inequality (2.2) continues to hold in the extended region.

Assume now that the operator $\tau_{\mathbf{e}_{j}}$ is weak mixing on $\Omega$ for some $j, 1 \leq j \leq d$, and let $\mathcal{E}_{j, \xi}(\Omega)=\left\{\partial_{j, \xi} g(\cdot): g \in \mathcal{H}(\Omega)\right\}$. Then by [21] the closure of $\mathcal{E}_{j, \xi}(\Omega) \subset \mathcal{H}(\Omega)$ contains the orthogonal complement of the constants, i.e. $\{g \in \mathcal{H}(\Omega):\langle g(\cdot)\rangle=$ $0\} \subset \overline{\mathcal{E}}_{j, \xi}(\Omega)$. Suppose now $g \in \mathcal{H}(\Omega)$ and $\langle g(\cdot)\rangle=0$. Then for any $\varepsilon>0$ there exists $\delta>0$ depending only on $\varepsilon, \xi$ and $g(\cdot)$, but not on $\eta>0$, such that

$$
\left\|T_{\xi^{\prime}, \eta} g-T_{\xi, \eta} g\right\|<\varepsilon,
$$

provided $\left|\xi^{\prime}-\xi\right|<\delta$. To see this first note that there exists $g_{\varepsilon, \xi} \in \mathcal{E}_{j, \xi}(\Omega)$ which satisfies $\left\|g-\partial_{j, \xi} g_{\varepsilon, \xi}\right\|<\varepsilon / 3$. Next observe that

$\left\|T_{\xi^{\prime}, \eta} \partial_{j, \xi} g_{\varepsilon, \xi}-T_{\xi, \eta} \partial_{j, \xi} g_{\varepsilon, \xi}\right\| \leq\left\|T_{\xi^{\prime}, \eta} \partial_{j, \xi^{\prime}} g_{\varepsilon, \xi}-T_{\xi, \eta} \partial_{j, \xi} g_{\varepsilon, \xi}\right\|+C\left|\xi^{\prime}-\xi\right|\left\|g_{\varepsilon, \xi}\right\|$,

where the constant $C$ depends only on $d$. It also follows from (2.17) that there is a constant $C$ depending only on $d$ such that

$$
\left\|T_{\xi^{\prime}, \eta} \partial_{j, \xi^{\prime}} g_{\varepsilon, \xi}-T_{\xi, \eta} \partial_{j, \xi} g_{\varepsilon, \xi}\right\| \leq C\left|\xi^{\prime}-\xi\right|^{1 / 2}\left\|g_{\varepsilon, \xi}\right\| \text {. }
$$

The inequality (2.21) follows from (2.22), (2.23) by choosing $\delta$ sufficiently small independent of $\eta>0$.

Since $\langle P \mathbf{b}(\cdot)\rangle=0$, the continuity of the function $h_{1}(\xi, \eta)$ in the region $\xi \in$ $\mathbf{R}^{d}, \eta \geq 0$, immediately follows from (2.21). The continuity of the functions $h_{m}(\cdot, \cdot), m \geq 1$, follow similarly by using the uniform bound $\left\|T_{\xi, \eta}\right\| \leq 1, \xi \in$ $\mathbf{R}^{d}, \eta \geq 0$.

Remark 1. Note that the projection operator $P$ in the formula (2.19) plays a critical role in establishing continuity. For a constant function $g(\cdot) \equiv v \in \mathbf{C}^{d}$, one has

$$
T_{\xi, \eta} g(\cdot)=\left[e(\xi)^{*} v\right] e(\xi) /\left[\eta / \Lambda+e(\xi)^{*} e(\xi)\right],
$$

which does not extend to a continuous function of $(\xi, \eta)$ on the set $\xi \in \mathbf{R}^{d}, \eta \geq 0$.

Next we show that the function $q(\xi, \eta)$ with domain $\xi \in \mathbf{R}^{d}, \eta>0$, can be extended to complex $\xi=\Re \xi+i \Im \xi \in \mathbf{C}^{d}$ with a small imaginary part.

Lemma 2.1. For fixed $\eta$ satisfying $0<\eta \leq \Lambda$, the $C^{\infty}$ operator valued function $\xi \rightarrow T_{\xi, \eta}$ from $\mathbf{R}^{d}$ to the space of bounded linear operators $\mathcal{B}[\mathcal{H}(\Omega)]$ on $\mathcal{H}(\Omega)$ has an analytic continuation to a region $\left\{\xi \in \mathbf{C}^{d}:|\Im \xi|<C_{1} \sqrt{\eta / \Lambda}\right\}$, where $C_{1}$ is a constant depending only on $d$. For $\xi$ in this region the norm of $T_{\xi, \eta}$ satisfies the inequality $\left\|T_{\xi, \eta}\right\| \leq 1+C_{2}|\Im \xi|^{2} /[\eta / \Lambda]$, where the constant $C_{2}$ depends only on $d$. 
Proof. The fact that there is an analytic continuation to the region $\left\{\xi \in \mathbf{C}^{d}:|\Im \xi|<\right.$ $\left.C_{1} \sqrt{\eta / \Lambda}\right\}$ is a consequence of the bound on the function $G_{\eta}(\cdot)$ of (2.8),

$$
\left|\nabla \nabla^{*} G_{\eta}(x)\right| \leq \frac{C_{3} \exp \left[-C_{4} \sqrt{\eta}|x|\right]}{[1+|x|]^{d}}, \quad x \in \mathbf{Z}^{d}, 0<\eta \leq 1,
$$

where the constants $C_{3}, C_{4}$ depend only on $d$. The bound on $\left\|T_{\xi, \eta}\right\|$ can be obtained from (2.6). Thus multiplying (2.6) by $\bar{\psi}(\xi, \eta, \omega)$, taking the expectation and using the Schwarz inequality, we see that

$$
\begin{aligned}
& \left\{1-C_{5}|\Im \xi|^{2} /[\eta / \Lambda]\right\}\left\|\partial_{\Re \xi} \psi(\xi, \eta, \cdot)\right\|^{2}+\frac{\eta}{2 \Lambda}\|\psi(\xi, \eta, \cdot)\|^{2} \\
& \leq\left\{\frac{1}{2}+C_{6}|\Im \xi|^{2} /[\eta / \Lambda]\right\}\|g\|^{2}+\frac{1}{2}\left\|\partial_{\Re \xi} \psi(\xi, \eta, \cdot)\right\|^{2}+\frac{\eta}{4 \Lambda}\|\psi(\xi, \eta, \cdot)\|^{2},
\end{aligned}
$$

where the constants $C_{5}, C_{6}$ depend only on $d$. Evidently (2.26) yields the bound on $\left\|T_{\xi, \eta}\right\|$ by taking $C_{1}$ sufficiently small, depending only on $d$.

Corollary 2.1. For fixed $\eta$ satisfying $0<\eta \leq \Lambda$, the $d \times d$ matrix function $q(\xi, \eta)$ with domain $\xi \in \mathbf{R}^{d}$ has an analytic continuation to a region $\left\{\xi \in \mathbf{C}^{d}:|\Im \xi|<\right.$ $\left.C_{1} \sqrt{\lambda \eta / \Lambda^{2}}\right\}$, where $C_{1}$ is a constant depending only on $d$. There is a constant $C_{2}$ depending only on $d$ such that for $\xi$ in this region,

$$
\|q(\xi, \eta)-q(\Re \xi, \eta)\| \leq \frac{C_{2} \Lambda^{2}}{\lambda} \frac{|\Im \xi|}{\sqrt{\eta / \Lambda}} .
$$

Proof. The fact that $q(\xi, \eta)$ has an analytic continuation follows from the representations (2.19), (2.20), Lemma 2.1 and the matrix norm bound $\|\mathbf{b}(\omega)\| \leq$ $1-\lambda / \Lambda, \omega \in \Omega$. On summing the perturbation series (2.20), we conclude that for $\xi$ satisfying $|\Im \xi|<C_{1} \sqrt{\lambda \eta / \Lambda^{2}}$, then $\|q(\xi, \eta)\| \leq C_{2} \Lambda^{2} / \lambda$ for a constant $C_{2}$ depending only on $d$, provided $C_{1}$ is chosen sufficiently small, depending only on $d$. By arguing as in Lemma 2.1 we also see that there are positive constants $C_{1}, C_{2}$ such that

$$
\left\|T_{\xi, \eta}-T_{\Re \xi, \eta}\right\| \leq C_{2}|\Im \xi| / \sqrt{\eta / \Lambda}, \quad \xi \in \mathbf{C}^{d},|\Im \xi|<C_{1} \sqrt{\eta / \Lambda} .
$$

The inequality (2.27) follows from (2.28).

We have seen in Corollary 2.1 that the periodic matrix function $q(\xi+i a, \eta), \xi \in$ $[-\pi, \pi]^{d}$, is bounded provided $a \in \mathbf{R}^{d}$ satisfies $|a| \leq C_{1} \sqrt{\lambda \eta / \Lambda^{2}}$. It was shown in [6] that derivatives of this function are in certain weak $L^{p}$ spaces. For $1 \leq p<\infty$ the weak $L^{p}$ space $L_{w}^{p}\left([-\pi, \pi]^{d}\right)$ is defined to be all measurable functions $f:[-\pi, \pi]^{d} \rightarrow$ C such that

$$
\operatorname{meas}\left\{\xi \in[-\pi, \pi]^{d}:|f(\xi)|>\mu\right\} \leq C^{p} / \mu^{p}, \quad \mu>0 .
$$

The weak $L^{p}$ norm of $f(\cdot),\|f\|_{p, w}$ is the minimum constant $C$ such that (2.29) holds. From [6] we have the following:

Proposition 2.2. Let $d \geq 1,0<\eta \leq \Lambda, 1 \leq k, k^{\prime} \leq d$, and $m=\left(m_{1}, \ldots, m_{d}\right)$ be a $d$-tuple of nonnegative integers with norm $|m|=m_{1}+\cdots+m_{d}$. Then there exists a positive constant $C_{1}$ depending only on $d$ such that if $|a| \leq C_{1} \sqrt{\lambda \eta / \Lambda^{2}}$ and $|m|<d$, the function

$$
\prod_{j=1}^{d}\left(\frac{\partial}{\partial \xi_{j}}\right)^{m_{j}} q_{k, k^{\prime}}(\xi+i a, \eta), \quad \xi \in[-\pi, \pi]^{d}
$$


is in the space $L_{w}^{p}\left([-\pi, \pi]^{d}\right)$ with $p=d /|m|$ and its norm is bounded by $C \Lambda$, where the constant $C$ depends only on $d$ and $\Lambda / \lambda \geq 1$.

If $|m|=d-1$ and $0<\delta \leq 1$, then for any $\rho \in \mathbf{R}^{d}$ satisfying $|\rho| \leq 1$, the function $(2.31)$

$$
\prod_{j=1}^{d}\left(\frac{\partial}{\partial \xi_{j}}\right)^{m_{j}}\left[q_{k, k^{\prime}}(\xi+\rho+i a, \eta)-q_{k, k^{\prime}}(\xi+i a, \eta)\right] /|\rho|^{1-\delta}, \quad \xi \in[-\pi, \pi]^{d},
$$

is in the space $L_{w}^{p}\left([-\pi, \pi]^{d}\right)$ with $p=d /(d-\delta)$ and its norm is bounded by $C_{\delta} \Lambda$, where the constant $C_{\delta}$ depends only on $d, \Lambda / \lambda$ and $\delta>0$.

\section{Configuration space estimates from Fourier space estimates}

In this section we shall show how to obtain configuration space estimates on $G_{\mathbf{a}, \eta}(x), x \in \mathbf{Z}^{d}$, from Fourier space estimates on the function $q(\xi, \eta), \xi \in \mathbf{R}^{d}$. In [6] it was shown that for $d \geq 3$, Proposition 2.2 implies the inequality

$$
0 \leq G_{\mathbf{a}, \eta}(x) \leq \frac{C \exp [-\gamma|x| \sqrt{\eta / \Lambda}]}{\Lambda(|x|+1)^{d-2}}, \quad x \in \mathbf{Z}^{d}, 0<\eta \leq \Lambda,
$$

where the positive constants $C, \gamma$ depend only on $d$ and $\Lambda / \lambda$. It was also shown that for $d \geq 2$, Proposition 2.2 implies a similar inequality for the gradient of $G_{\mathbf{a}, \eta}(x)$,

$$
\left|\nabla G_{\mathbf{a}, \eta}(x)\right| \leq \frac{C \exp [-\gamma|x| \sqrt{\eta / \Lambda}]}{\Lambda(|x|+1)^{d-1}}, \quad x \in \mathbf{Z}^{d}, 0<\eta \leq \Lambda .
$$

Finally for $d \geq 1$, Proposition 2.2 implies Hölder continuity of $\nabla G_{\mathbf{a}, \eta}(x)$,

$$
\begin{gathered}
\left|\nabla G_{\mathbf{a}, \eta}\left(x^{\prime}\right)-\nabla G_{\mathbf{a}, \eta}(x)\right| \leq\left|x^{\prime}-x\right|^{1-\delta} \frac{C_{\delta} \exp [-\gamma|x| \sqrt{\eta / \Lambda}]}{\Lambda(|x|+1)^{d-\delta}}, \\
0<\eta \leq \Lambda, \quad x^{\prime}, x \in \mathbf{Z}^{d}, 1 / 2 \leq\left(\left|x^{\prime}\right|+1\right) /(|x|+1) \leq 2,
\end{gathered}
$$

for any $\delta, 0<\delta \leq 1$, where the constant $C_{\delta}$ now depends on $\delta>0$ as well as on $d$ and $\Lambda / \lambda$. Here we assume a strengthened version of Proposition 2.2 (Hypothesis 3.1 below), and show how it implies the inequalities (1.10), (1.11) and almost implies (1.12). In subsequent sections we shall establish Hypothesis 3.1 for the environments $(\Omega, \mathcal{F}, P)$ introduced in $\S 1$.

Hypothesis 3.1. There exist positive constants $C_{1}, C_{2}$ and $\alpha \leq 1$ depending only on $d$ and $\Lambda / \lambda$, such that

$$
\begin{aligned}
& \left\|q\left(\xi^{\prime}, \eta^{\prime}\right)-q(\xi, \eta)\right\| \leq C_{1} \Lambda\left[\left|\xi^{\prime}-\xi\right|^{\alpha}+\left|\left(\eta^{\prime}-\eta\right) / \Lambda\right|^{\alpha / 2}\right] \\
& 0<\eta \leq \eta^{\prime} \leq \Lambda, \quad \xi^{\prime}, \xi \in \mathbf{C}^{d} \text { with }|\Im \xi|+\left|\Im \xi^{\prime}\right| \leq C_{2} \sqrt{\eta / \Lambda} .
\end{aligned}
$$

With the same assumptions as in Proposition 2.2, the derivative (2.30) is in the space $L_{w}^{p}\left([-\pi, \pi]^{d}\right)$ with $p=d /(|m|-\alpha)$ and its norm is bounded by $C \Lambda$, where the constant $C$ depends only on $d$ and $\Lambda / \lambda$. The difference in (2.31) is in the space $L_{w}^{p}\left([-\pi, \pi]^{d}\right)$ with $p=d /(d-\delta-\alpha)$ and its norm is bounded by $C \Lambda$, where now $\alpha$ and $C$ depend on $\delta$ as well as $d$ and $\Lambda / \lambda$.

Hypothesis 3.1 enables us to compare the function $G_{\mathbf{a}, \eta}(x), x \in \mathbf{Z}^{d}$, to the function $G_{\mathbf{a}_{\text {hom }}, \eta}^{\text {lattice }}(x), x \in \mathbf{Z}^{d}$, defined by

$$
G_{\mathbf{a}_{\text {hom }}, \eta}^{\text {lattice }}(x)=\frac{1}{(2 \pi)^{d}} \int_{[-\pi, \pi]^{d}} \frac{e^{-i \xi \cdot x}}{\eta+e(\xi)^{*} q(0,0) e(\xi)} d \xi
$$


Theorem 3.1. Assume the function $q(\cdot, \cdot)$ in (2.1) satisfies Hypothesis 3.1 , and $\eta$ lies in the interval $0<\eta \leq \Lambda$. Then there exist positive constants $\alpha, \gamma, C$ with $\alpha \leq 1$, depending only on $d$ and $\Lambda / \lambda$ such that

$$
\begin{aligned}
\left|G_{\mathbf{a}, \eta}(x)-G_{\mathbf{a}_{\text {hom }}, \eta}^{\text {lattice }}(x)\right| & \leq \frac{C}{\Lambda(|x|+1)^{d-2+\alpha}} e^{-\gamma \sqrt{\eta / \Lambda}|x|}, \quad x \in \mathbf{Z}^{d}, \\
\left|\nabla G_{\mathbf{a}, \eta}(x)-\nabla G_{\mathbf{a}_{\text {hom }}, \eta}^{\text {lattice }}(x)\right| & \leq \frac{C}{\Lambda(|x|+1)^{d-1+\alpha}} e^{-\gamma \sqrt{\eta / \Lambda}|x|}, \quad x \in \mathbf{Z}^{d},
\end{aligned}
$$

where (3.6) holds if $d \geq 2$ and (3.7) if $d \geq 1$. For $d \geq 1$ and $\delta$ satisfying $0<\delta \leq 1$, there is the inequality

$$
\leq\left|x^{\prime}-x\right|^{1-\delta} \frac{C}{\Lambda(|x|+1)^{d-\delta+\alpha}} e^{-\gamma \sqrt{\eta / \Lambda}|x|}, \quad x^{\prime}, x \in \mathbf{Z}^{d}, 1 / 2 \leq\left(\left|x^{\prime}\right|+1\right) /(|x|+1) \leq 2,
$$

where $C$ and $\alpha$ in (3.8) depend on $\delta>0$ as well as $d$ and $\Lambda / \lambda$. In particular, $\alpha<\delta$.

Proof. Let $\tilde{G}_{\mathbf{a}_{\text {hom }, \eta}}(x)$ be the function

$$
\tilde{G}_{\mathbf{a}_{\mathrm{hom}, \eta}}(x)=\frac{1}{(2 \pi)^{d}} \int_{[-\pi, \pi]^{d}} \frac{e^{-i \xi \cdot x}}{\eta+e(\xi)^{*} q(0, \eta) e(\xi)} d \xi,
$$

so (2.1) and Corollary 2.1 imply that

$$
G_{\mathbf{a}, \eta}(x)-\tilde{G}_{\mathbf{a}_{\mathrm{hom}}, \eta}(x)=\frac{e^{a . x}}{(2 \pi)^{d}} \int_{[-\pi, \pi]^{d}} e^{-i \xi \cdot x} f(\xi) d \xi,
$$

where the function $f(\xi)$ is given by the formula

$$
f(\xi)=\frac{e(\xi-i a)^{*}\{q(0, \eta)-q(\xi+i a, \eta)\} e(\xi+i a)}{\left[\eta+e(\xi-i a)^{*} q(0, \eta) e(\xi+i a)\right]\left[\eta+e(\xi-i a)^{*} q(\xi+i a, \eta) e(\xi+i a)\right]} .
$$

It follows from (3.4) and Corollary 2.1 that there are positive constants $C_{1}, C_{2}$ depending only on $d$ and $\Lambda / \lambda$ such that the function in (3.11) is bounded by

$$
|f(\xi)| \leq \frac{C_{1}}{\Lambda\left[\eta / \Lambda+|e(\xi)|^{2}\right]^{1-\alpha / 2}}, \quad \text { for }|a| \leq C_{2} \sqrt{\eta / \Lambda} .
$$

Choosing $a$ appropriately and $\alpha<1$ one sees already from (3.10), (3.12) that if $d=1$, the function $\left|G_{\mathbf{a}, \eta}(x)-\tilde{G}_{\mathbf{a}_{\mathrm{hom}, \eta}}(x)\right|$ is bounded by the RHS of (3.6) provided $\sqrt{\eta / \Lambda}|x| \geq 1$.

We wish to show for $d=1$ that $\left|\nabla G_{\mathbf{a}, \eta}(x)-\nabla \tilde{G}_{\mathbf{a}_{\mathrm{hom}}, \eta}(x)\right|$ is bounded by the RHS of (3.7). The representation of $\nabla G_{\mathbf{a}, \eta}(x)-\nabla \tilde{G}_{\mathbf{a}_{\mathrm{hom}}, \eta}(x)$ corresponding to (3.10) is

$$
\nabla G_{\mathbf{a}, \eta}(x)-\nabla \tilde{G}_{\mathbf{a}_{\mathrm{hom}, \eta}}(x)=\frac{e^{a \cdot x}}{(2 \pi)^{d}} \int_{[-\pi, \pi]^{d}} e^{-i \xi \cdot x} e(\xi) f(\xi) d \xi .
$$

Let $\rho \in \mathbf{R}^{d}$ be the vector of minimum norm which satisfies $e^{-i \rho . x}=-1$. Then we have that

$$
\int_{[-\pi, \pi]^{d}} e^{-i \xi \cdot x} e(\xi) f(\xi) d \xi\left|\leq \frac{1}{2} \int_{[-\pi, \pi]^{d}}\right| e(\xi) f(\xi)-e(\xi+\rho) f(\xi+\rho) \mid d \xi .
$$

The integral on the RHS of (3.14) can be estimated by separately estimating the integral over the region $|\xi|<C /[|x|+1]$ and $|\xi|>C /[|x|+1]$ for a sufficiently large 
universal constant $C$. Using the estimate for $|f(\xi)|$ obtained from (3.12) and the fact that $|\rho| \leq C^{\prime} /[|x|+1]$, we see that the integral over $|\xi|<C /[|x|+1]$ is bounded by a constant times $[|x|+1]^{-\alpha}$. To estimate the integral over $|\xi|>C /[|x|+1]$ we use the Hölder continuity of the function $f(\cdot)$. From (3.4) it follows that

$$
|e(\xi) f(\xi)-e(\xi+\rho) f(\xi+\rho)| \leq \frac{C_{1}|\rho|^{\alpha}}{\Lambda\left[\eta / \Lambda+|e(\xi)|^{2}\right]^{1 / 2}}, \quad|\xi|>C /[|x|+1],
$$

for a constant $C_{1}$ depending only on $d$ and $\Lambda / \lambda$, provided $C$ is sufficiently large. Hence the integral over $|\xi|>C /[|x|+1]$ is bounded by a constant times $[|x|+$ $1]^{-\alpha} \log [|x|+2]$. We have shown that $\left|\nabla G_{\mathbf{a}, \eta}(x)-\nabla \tilde{G}_{\mathbf{a h o m}, \eta}(x)\right|$ is bounded by the RHS of (3.7) for any $\alpha$ less than the Hölder constant in (3.4). We can similarly bound $\left|\nabla \tilde{G}_{\mathbf{a}_{\text {hom }}, \eta}(x)-\nabla G_{\mathbf{a}_{\text {hom }}, \eta}^{\text {lattice }}(x)\right|$ by the RHS of (3.7) by using the estimate $\|q(0, \eta)-q(0,0)\| \leq C \Lambda(\eta / \Lambda)^{\alpha / 2}$ from (3.4). Hence (3.7) holds for $d=1$, and by similar argument (3.8).

In order to prove (3.6) for $d \geq 2$ we need to use the bounds on the derivatives of the function $q(\cdot, \cdot)$ given in Proposition 2.2. For $d=2$ the first derivative estimate is sufficient. Thus we write

$$
\begin{aligned}
& \text { (3.16) } \int_{[-\pi, \pi]^{d}} e^{-i \xi \cdot x} f(\xi) d \xi=\int_{|\xi|<C /[|x|+1]} e^{-i \xi \cdot x} f(\xi) d \xi \\
& +\frac{i}{|x|^{2}} \int_{|\xi|=C /[|x|+1]}[x \cdot \mathbf{n}(\xi)] e^{-i \xi \cdot x} f(\xi) d \xi-\frac{i}{|x|^{2}} \int_{|\xi|>C /[|x|+1]} e^{-i \xi \cdot x}\left[x \cdot \nabla_{\xi} f(\xi)\right] d \xi,
\end{aligned}
$$

where $\mathbf{n}(\xi)$ is the unit inward normal vector at $\xi$ on the sphere $\{|\xi|=C /[|x|+1]\}$. It follows from (3.12) that the first two integrals on the RHS of (3.16) are bounded by $C_{2} / \Lambda[|x|+1]^{d-2+\alpha}$ for some constant $C_{2}$ depending only on $d$ and $\Lambda / \lambda$. The third integral can be similarly bounded for $d=2$ by using the fact from Hypothesis 3.1 that the function $\nabla_{\xi} q(\xi+i a, \eta)$ is in $L_{w}^{p}\left([-\pi, \pi]^{2}\right)$ with $p=2 /(1-\alpha)$. To see this we note that for any measure space $(X, \mathcal{B}, \mu)$ and $1<p<\infty$ one has that if $g \in L_{w}^{p}(X)$, then

$$
\int_{E}|g| d \mu \leq C_{p}\|g\|_{p, w} m(E)^{1-1 / p}, \quad E \in \mathcal{B},
$$

where $C_{p}$ depends only on $p$. It follows that for $n=1,2, \ldots$,

$$
\int_{2^{n-1} C /[|x|+1]<|\xi|<2^{n} C /[|x|+1]}\left|\nabla_{\xi} f(\xi)\right| d \xi \leq \frac{C_{1} 2^{-(1-\alpha) n}}{\Lambda[|x|+1]^{\alpha-1}},
$$

where $C_{1}$ depends only on $d=2$ and $\Lambda / \lambda$. Hence by summing over $n \geq 1$ in (3.18) we see that for $d=2$ the function $\left|G_{\mathbf{a}, \eta}(x)-\tilde{G}_{\mathbf{a}_{\text {hom }}, \eta}(x)\right|$ is bounded by the RHS of (3.6). We can bound $\left|\tilde{G}_{\mathrm{ahom}, \eta}(x)-G_{\mathrm{a}_{\mathrm{hom}, \eta}}^{\text {lattice }}(x)\right|$ using the Hölder continuity (3.4) of $q(0, \eta), 0 \leq \eta \leq \Lambda$, by the RHS of (3.6) for any $\alpha$ smaller than the Hölder constant in (3.4). We have therefore proven (3.6) for $d=2$.

To prove (3.7) for $d=2$ we need to use the fact from Hypothesis 3.1 that the difference $\left[\nabla_{\xi} q(\xi+\rho+i a, \eta)-\nabla_{\xi} q(\xi+i a, \eta)\right] /|\rho|^{1-\delta}$ is in $L_{w}^{p}\left([-\pi, \pi]^{2}\right)$ with $p=2 /(2-\delta-\alpha)$ and norm bounded independent of $|\rho| \leq 1$. Thus in bounding $\nabla G_{\mathbf{a}, \eta}(x)-\nabla \tilde{G}_{\mathbf{a}_{\text {hom }, \eta}}(x)$ we write the integral over $\xi$ as in (3.16). The first two terms on the RHS can be bounded in a straightforward way. To bound the third term we need to estimate the integral 


$$
\begin{aligned}
& \int_{|\xi|>C /[|x|+1]} e^{-i \xi \cdot x}\left(x . \nabla_{\xi}\right)[e(\xi) f(\xi)] d \xi \\
= & \frac{1}{2} \int_{|\xi|>C^{\prime} /[|x|+1]} e^{-i \xi \cdot x}\left(x \cdot \nabla_{\xi}\right)[e(\xi) f(\xi)-e(\xi+\rho) f(\xi+\rho)] d \xi+\text { Error. }
\end{aligned}
$$

In (3.19) the vector $\rho \in \mathbf{R}^{d}$ is as in (3.14) and $C^{\prime}$ is a universal constant. The error term in (3.19) is bounded by

$$
\text { Error } \leq \int_{C / 10[|x|+1]<|\xi|<10 C /[|x|+1]}\left|\left(x . \nabla_{\xi}\right)[e(\xi) f(\xi)]\right| d \xi,
$$

which can be appropriately estimated by using the fact that $\nabla_{\xi} q(\xi+i a, \eta)$ is in $L_{w}^{p}\left([-\pi, \pi]^{2}\right)$ with $p=2 /(1-\alpha)$. Similar to (3.18) we have for $n=1,2, \ldots$ the bound

$$
\begin{aligned}
& \int_{2^{n-1} C^{\prime} /[|x|+1]<|\xi|<2^{n} C^{\prime} /[|x|+1]}\left|\nabla_{\xi}[e(\xi) f(\xi)-e(\xi+\rho) f(\xi+\rho)]\right| d \xi \\
& \leq \frac{C_{\delta} 2^{-(1-\alpha-\delta) n}|\rho|^{1-\delta}}{\Lambda[|x|+1]^{\alpha+\delta-1}}
\end{aligned}
$$

where $C_{\delta}$ depends only on $d=2$ and $\Lambda / \lambda$. Now choosing $\alpha<1-\delta$ in (3.21), we conclude that $\left|\nabla G_{\mathbf{a}, \eta}(x)-\nabla \tilde{G}_{\mathbf{a}_{\mathrm{hom}, \eta}}(x)\right|$ is bounded by the RHS of (3.7). As in the previous paragraph, we can bound $\left|\nabla \tilde{G}_{\mathbf{a}_{\text {hom }, \eta}}(x)-\nabla G_{\mathbf{a}_{\text {hom }}, \eta}^{\text {lattice }}(x)\right|$ using the Hölder continuity (3.4) of $q(0, \eta), 0 \leq \eta \leq \Lambda$, by the RHS of (3.7) for any $\alpha$ smaller than the Hölder constant in (3.4). We have therefore proven (3.7) for $d=2$.

We proceed similarly for the proof of (3.8) in the case $d=2$. Thus we write

$$
\begin{aligned}
& \int_{|\xi|>C /[|x|+1]}\left[e^{-i \xi \cdot x}-e^{-i \xi \cdot x^{\prime}}\right]\left(x \cdot \nabla_{\xi}\right)[e(\xi) f(\xi)] d \xi \\
& =\frac{1}{2} \int_{|\xi|>C^{\prime} /[|x|+1]}\left[e^{-i \xi \cdot x}-e^{-i \xi \cdot x^{\prime}}\right]\left(x \cdot \nabla_{\xi}\right)[e(\xi) f(\xi)-e(\xi+\rho) f(\xi+\rho)] d \xi \\
& +\frac{1}{2}\left[1-e^{i \rho \cdot\left(x^{\prime}-x\right)}\right] \int_{|\xi|>C^{\prime} /[|x|+1]} e^{-i \xi \cdot x}\left(x \cdot \nabla_{\xi}\right)[e(\xi+\rho) f(\xi+\rho)] d \xi+\text { Error, }
\end{aligned}
$$

where $\rho \in \mathbf{R}^{d}$ is the vector of minimum norm such that $e^{-i \rho \cdot x^{\prime}}=-1$. Arguing as in (3.20) we see that

$$
\text { | Error }\left|\leq C_{1}\right| x-x^{\prime} \mid / \Lambda[|x|+1]^{\alpha},
$$

where $C_{1}$ depends only on $\Lambda / \lambda$. From (3.21) we see that the first term on the RHS of (3.22) is bounded by the sum

$$
|x|\left|x-x^{\prime}\right|^{1-\delta} \sum_{n=1}^{\infty}\left(\frac{2^{n} C^{\prime}}{[|x|+1]}\right)^{1-\delta} \frac{C_{\delta^{\prime}} 2^{-\left(1-\alpha-\delta^{\prime}\right) n}|\rho|^{1-\delta^{\prime}}}{\Lambda[|x|+1]^{\alpha+\delta^{\prime}-1}},
$$

for any $\delta^{\prime}$ satisfying $0<\delta^{\prime} \leq 1$. Provided $\alpha<\delta$ we may choose $\delta^{\prime}>0$ so that the sum in (3.24) converges, whence the sum is bounded by $C_{\delta}\left|x-x^{\prime}\right|^{1-\delta} / \Lambda[|x|+1]^{\alpha-\delta}$ for a constant $C_{\delta}$ depending only on $\delta>0$ as well as $\Lambda / \lambda$. The second term on the RHS of (3.22) is $\left[1-e^{i \rho .\left(x^{\prime}-x\right)}\right]$ times an integral similar to the integral on the LHS of (3.19). Hence the second term is bounded in absolute value by $C_{1}\left|x-x^{\prime}\right| / \Lambda[|x|+1]^{\alpha}$, where $C_{1}$ depends only on $\Lambda / \lambda$. We have therefore shown 
that $\left|\nabla G_{\mathbf{a}, \eta}(x)-\nabla \tilde{G}_{\mathbf{a}_{\mathrm{hom}, \eta}}(x)\right|$ is bounded by the RHS of (3.8). Since we can argue as previously to bound $\left|\nabla \tilde{G}_{\mathbf{a}_{\mathrm{hom}, \eta}}(x)-\nabla G_{\mathbf{a}_{\mathrm{hom}, \eta}}^{\text {lattice }}(x)\right|$, the proof of (3.8) is complete.

To prove the result for $d \geq 3$ we use multiple integration by parts and the integrability properties of the higher derivatives of $q(\xi+i a, \eta), \xi \in[-\pi, \pi]^{d}$, given in Hypothesis 3.1.

Finally we compare the function $G_{\mathbf{a}_{\text {hom }}, \eta}^{\text {latice }}(\cdot)$ of (3.5) to the Green's function

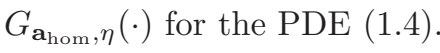

Lemma 3.1. Assume $\eta$ lies in the interval $0<\eta \leq \Lambda$. Then there exist positive constants $\gamma, C$ depending only on $d$ and $\Lambda / \lambda$ such that

$$
\begin{gathered}
\left|G_{\mathbf{a}_{\mathrm{hom}, \eta}}^{\text {lattice }}(x)-G_{\mathbf{a}_{\mathrm{hom}}, \eta}(x)\right| \leq \frac{C}{\Lambda(|x|+1)^{d-1}} e^{-\gamma \sqrt{\eta / \Lambda}|x|}, \quad x \in \mathbf{Z}^{d}-\{0\}, \\
\left|\nabla G_{\mathbf{a}_{\mathrm{hom}, \eta}}^{\text {lattice }}(x)-\nabla G_{\mathbf{a}_{\mathrm{hom}}, \eta}(x)\right| \leq \frac{C}{\Lambda(|x|+1)^{d}} e^{-\gamma \sqrt{\eta / \Lambda}|x|}, \quad x \in \mathbf{Z}^{d}-\{0\},
\end{gathered}
$$

$$
\left|\nabla \nabla G_{\mathbf{a}_{\text {hom }}, \eta}^{\text {lattice }}(x)-\nabla \nabla G_{\mathbf{a}_{\text {hom }}, \eta}(x)\right| \leq \frac{C}{\Lambda(|x|+1)^{d+1}} e^{-\gamma \sqrt{\eta / \Lambda}|x|}, \quad x \in \mathbf{Z}^{d}-\{0\},
$$

where (3.25) holds if $d \geq 2$ and (3.26), (3.27) if $d \geq 1$.

Proof. Taking $\mathbf{a}_{\text {hom }}=q(0,0)$ in (1.4), we see from (3.5) that $G_{\mathbf{a}_{\text {hom }}, \eta}^{\text {latice }}(\cdot)$ is the Green's function for the discrete elliptic equation on $\mathbf{Z}^{d}$ corresponding to (1.4). To prove the theorem we follow a standard method of numerical analysis for estimating the error between the solution of a continuous problem and its approximating discrete problems. The method is to regard the solution of the continuous problem as an approximate solution to the discrete problem. An alternative approach based on comparison of the Fourier representation (3.5) of the lattice Green's function $G_{\mathbf{a}_{\text {hom }}, \eta}^{\text {lattice }}(\cdot)$ to the Fourier representation of the continuous Green's function $G_{\mathbf{a}_{\text {hom }, \eta}(\cdot)}$ is pursued in [16].

Let $f: \mathbf{R}^{d} \rightarrow \mathbf{R}$ be a nonnegative $C^{\infty}$ function with support contained in the ball $\left\{x \in \mathbf{R}^{d}:|x|<1\right\}$ and $u(\cdot, \eta)$ be the solution to the PDE (1.4). With $\nabla_{x}, \nabla_{x}^{*}$ denoting the discrete operators (1.3), we have that

$$
\begin{array}{r}
\eta u(x+z, \eta)+\nabla_{x}^{*} \mathbf{a}_{\mathrm{hom}} \nabla_{x} u(x+z, \eta)=\eta u(x+z, \eta)+\text { Trace }\left[\mathbf{a}_{\mathrm{hom}} A(x+z)\right], \\
x \in \mathbf{Z}^{d}, z \in \mathbf{R}^{d},
\end{array}
$$

where the $d \times d$ matrix $A(y)=\left[A_{i, j}(y)\right], y \in \mathbf{R}^{d}$, is given by the formula

$$
A_{i, j}(y)=u(y)+u\left(y+\mathbf{e}_{j}-\mathbf{e}_{i}\right)-u\left(y+\mathbf{e}_{j}\right)-u\left(y-\mathbf{e}_{i}\right)=E\left[\frac{\partial^{2} u\left(y+Y_{i, j}, \eta\right)}{\partial y_{i} \partial y_{j}}\right],
$$

with $Y_{i, j}$ the random variable uniformly distributed in the unit square $\left\{y_{j} \mathbf{e}_{j}-y_{i} \mathbf{e}_{i} \in\right.$ $\left.\mathbf{R}^{d}: 0 \leq y_{i}, y_{j} \leq 1\right\}$. It follows from (1.4), (3.28), (3.29) that

(3.30) $\eta u(x+z, \eta)+\nabla_{x}^{*} \mathbf{a}_{\mathrm{hom}} \nabla_{x} u(x+z, \eta)=f(x+z)+h(x+z), \quad x \in \mathbf{Z}^{d}, z \in \mathbf{R}^{d}$, where the function $h: \mathbf{R}^{d} \rightarrow \mathbf{R}$ is given by the formula

$$
h(y)=\sum_{i, j=1}^{d} \mathbf{a}_{\mathrm{hom}}(i, j)\left\{E\left[\frac{\partial^{2} u\left(y+Y_{i, j}, \eta\right)}{\partial y_{i} \partial y_{j}}\right]-\frac{\partial^{2} u(y, \eta)}{\partial y_{i} \partial y_{j}}\right\}, \quad y \in \mathbf{R}^{d}
$$


We conclude that

$$
u(x+z, \eta)=\sum_{y \in \mathbf{Z}^{d}} G_{\mathbf{a}_{\text {hom }}, \eta}^{\text {latice }}(x-y)[f(y+z)+h(y+z)], \quad x \in \mathbf{Z}^{d}, z \in \mathbf{R}^{d} .
$$

Let $Q_{0} \subset \mathbf{R}^{d}$ be the unit cube centered at the origin. Then we have that

$$
\begin{aligned}
\int_{Q_{0}} d z[u(x+z, \eta) & \left.-\sum_{y \in \mathbf{Z}^{d}} G_{\mathbf{a}_{\mathrm{hom}}, \eta}^{\text {lattice }}(x-y) f(y+z)\right] \\
& =\left[G_{\mathbf{a}_{\mathrm{hom}}, \eta}(x)-G_{\mathbf{a}_{\mathrm{hom}}, \eta}^{\text {lattice }}(x)\right] \int_{\mathbf{R}^{d}} f(y) d y+\operatorname{Error}(x)
\end{aligned}
$$

where $|\operatorname{Error}(x)|$ is bounded by the RHS of (3.25). Next we observe from (3.31) that

$$
\int_{Q_{0}} d z \sum_{y \in \mathbf{Z}^{d}} h(y+z)=0
$$

and hence (3.32) implies that the LHS of (3.33) is equal to

$$
\int_{Q_{0}} d z \sum_{y \in \mathbf{Z}^{d}}\left[G_{\mathbf{a}_{\mathrm{hom}}, \eta}^{\text {lattice }}(x-y)-G_{\mathbf{a}_{\mathrm{hom}, \eta}}^{\text {lattice }}(x)\right] h(y+z) .
$$

Using the fact that the distribution of $Y_{j, i}$ is the same as the distribution of $-Y_{i, j}$, we see from (3.31) that $h(\cdot)$ is bounded by the fourth derivative of $u(\cdot, \eta)$, whence we conclude that there is a constant $C$ such that

$$
|h(y)| \leq \frac{C}{\Lambda(|y|+1)^{d+2}} e^{-\gamma \sqrt{\eta / \Lambda}|y|}, \quad y \in \mathbf{R}^{d} .
$$

The inequality (3.25) follows from (3.33), (3.35), (3.36). The inequalities (3.26), (3.27) follow by similar argument.

Evidently the inequalities (3.6), (3.7) of Theorem 3.1 together with Lemma 3.1 imply the inequalities (1.10), (1.11).

\section{INDEPENDENT VARIABLE ENVIRONMENT}

Our goal in this section will be to prove Hypothesis 3.1 in the case when the variables $\mathbf{a}\left(\tau_{x} \cdot\right), x \in \mathbf{Z}^{d}$, are independent. Following [5] we first consider the case of a Bernoulli environment. Thus for each $n \in \mathbf{Z}^{d}$ let $Y_{n}$ be independent Bernoulli variables, whence $Y_{n}= \pm 1$ with equal probability. The probability space $(\Omega, \mathcal{F}, P)$ is then the space generated by all the variables $Y_{n}, n \in \mathbf{Z}^{d}$. A point $\omega \in \Omega$ is a set of configurations $\left\{\left(Y_{n}, n\right): n \in \mathbf{Z}^{d}\right\}$. For $y \in \mathbf{Z}^{d}$ the translation operator $\tau_{y}$ acts on $\Omega$ by taking the point $\omega=\left\{\left(Y_{n}, n\right): n \in \mathbf{Z}^{d}\right\}$ to $\tau_{y} \omega=\left\{\left(Y_{n+y}, n\right): n \in \mathbf{Z}^{d}\right\}$. The random matrix $\mathbf{a}(\cdot)$ is then defined by

$$
\mathbf{a}(\omega)=\left(1+\gamma Y_{0}\right) I_{d}, \quad \omega=\left\{\left(Y_{n}, n\right): n \in \mathbf{Z}^{d}\right\},
$$

where $0 \leq \gamma<1$. In $[5]$ we defined for $1 \leq p<\infty$ Fock spaces $\mathcal{F}^{p}\left(\mathbf{Z}^{d}\right)$ of complex valued functions, and observed that $\mathcal{F}^{2}\left(\mathbf{Z}^{d}\right)$ is unitarily equivalent to $L^{2}(\Omega)$. Thus for each $N=1,2, \ldots$, let $\mathbf{Z}^{d, N}$ be the collection of all sets of $N$ distinct elements 
$\left\{x_{1}, \ldots, x_{N}\right\}$ with $x_{j} \in \mathbf{Z}^{d}, 1 \leq j \leq N$. For $1 \leq p<\infty$ a function $\psi_{N}: \mathbf{Z}^{d, N} \rightarrow \mathbf{C}$ is in $L^{p}\left(\mathbf{Z}^{d, N}\right)$ with norm $\left\|\psi_{N}\right\|_{p}$ if

$$
\left\|\psi_{N}\right\|_{p}^{p}=\sum_{m \in \mathbf{Z}^{d, N}}\left|\psi_{N}(m)\right|^{p}<\infty .
$$

The Fock space $\mathcal{F}^{p}\left(\mathbf{Z}^{d}\right)$ consists of all sets $\psi=\left\{\psi_{N}: N=0,1,2, \ldots\right\}$ of functions $\psi_{N} \in L^{p}\left(\mathbf{Z}^{d, N}\right), N=1,2, \ldots$, and $\psi_{0} \in \mathbf{C}$ with norm $\|\psi\|_{p}$ satisfying

$$
\|\psi\|_{p}^{p}=\left|\psi_{0}\right|^{p}+\sum_{N=1}^{\infty}\left\|\psi_{N}\right\|_{p}^{p}<\infty .
$$

We can similarly define Fock spaces $\mathcal{H}_{\mathcal{F}}^{p}\left(\mathbf{Z}^{d}\right)$ of vector valued functions with domain $\mathbf{C}^{d}$, such that $\mathcal{H}_{\mathcal{F}}^{2}\left(\mathbf{Z}^{d}\right)$ is unitarily equivalent to $\mathcal{H}(\Omega)$. Hence we can regard the operator $T_{\xi, \eta}$ of (2.7) as acting on $\mathcal{H}_{\mathcal{F}}^{2}\left(\mathbf{Z}^{d}\right)$, and by unitary equivalence it is a bounded operator satisfying $\left\|T_{\xi, \eta}\right\| \leq 1$ for $\xi \in \mathbf{R}^{d}, \eta>0$. From (2.7) we see that the action of $T_{\xi, \eta}$ on a function $\psi=\left\{\psi_{N}: N=0,1,2, \ldots\right\}$ in $\mathcal{H}_{\mathcal{F}}^{2}\left(\mathbf{Z}^{d}\right)$ is given by the formula

$$
\begin{aligned}
& T_{\xi, \eta} \psi_{N}\left(x_{1}, \ldots, x_{N}\right) \\
& \quad=\sum_{x^{\prime} \in \mathbf{Z}^{d}}\left\{\nabla \nabla^{*} G_{\eta / \Lambda}\left(x^{\prime}\right)\right\}^{*} \exp \left[-i x^{\prime} \cdot \xi\right] \psi_{N}\left(x_{1}-x^{\prime}, \ldots, x_{N}-x^{\prime}\right), N \geq 1 .
\end{aligned}
$$

We may therefore apply the Calderon-Zygmund theorem [22] to conclude the following:

Lemma 4.1. For $\xi \in \mathbf{R}^{d}, 0<\eta \leq 1$, and $1<p<\infty$, the operator $T_{\xi, \eta}$ is a bounded operator on $\mathcal{H}_{\mathcal{F}}^{p}\left(\mathbf{Z}^{d}\right)$ with norm $\left\|T_{\xi, \eta}\right\|_{p}$ satisfying an inequality $\left\|T_{\xi, \eta}\right\|_{p} \leq$ $1+\delta(p)$, where $\lim _{p \rightarrow 2} \delta(p)=0$.

It is well known for the independent variable environment $(\Omega, \mathcal{F}, P)$ that the operators $\tau_{\mathbf{e}_{j}}, j=1, \ldots d$, are strong mixing on $\Omega$. Hence Proposition 2.1 implies that the function $q(\xi, \eta)$ with domain $\xi \in[-\pi, \pi]^{d}, 0<\eta \leq \Lambda$, is uniformly continuous. Lemma 4.1 enables us to improve this result to uniform Hölder continuity.

Proposition 4.1. The function $q(\xi, \eta)$ of (2.5) with domain $\xi \in[-\pi, \pi]^{d}, 0<$ $\eta \leq \Lambda$, is uniformly Hölder continuous. That is, there exist positive constants $C, \alpha$ with $0<\alpha \leq 1$ depending only on $d$ and $\Lambda / \lambda$, such that $\left\|q\left(\xi^{\prime}, \eta^{\prime}\right)-q(\xi, \eta)\right\| \leq$ $C \Lambda\left[\left|\xi^{\prime}-\xi\right|^{\alpha}+\left|\left(\eta^{\prime}-\eta\right) / \Lambda\right|^{\alpha / 2}\right]$ for $\xi^{\prime}, \xi \in[-\pi, \pi]^{d}$ and $0<\eta, \eta^{\prime} \leq \Lambda$.

Proof. We use the representation (2.19), (2.20) for $q(\xi, \eta)$. From (2.19) we have that

$$
\begin{aligned}
h_{m}\left(\xi^{\prime}, \eta\right) & -h_{m}(\xi, \eta) \\
= & \sum_{j=0}^{m-1}\left\langle\mathbf{b}(\cdot)\left[P T_{\xi^{\prime}, \eta} \mathbf{b}(\cdot)\right]^{m-1-j} P\left[T_{\xi^{\prime}, \eta}-T_{\xi, \eta}\right] \mathbf{b}(\cdot)\left[P T_{\xi, \eta} \mathbf{b}(\cdot)\right]^{j}\right\rangle .
\end{aligned}
$$

From (2.7) and the weak Young inequality we see that for $0<\alpha \leq 1$, the operator $P\left[T_{\xi^{\prime}, \eta}-T_{\xi, \eta}\right]$ from $\mathcal{H}_{\mathcal{F}}^{p}\left(\mathbf{Z}^{d}\right)$ to $\mathcal{H}_{\mathcal{F}}^{2}\left(\mathbf{Z}^{d}\right)$ with $p=2 d /(d+2 \alpha)$ is bounded with norm satisfying

$$
\left\|P\left[T_{\xi^{\prime}, \eta}-T_{\xi, \eta}\right]\right\|_{p, 2} \leq C\left|\xi^{\prime}-\xi\right|^{\alpha}
$$


for a constant $C$ depending only on $d$ if $d \geq 3$. In the case $d \leq 2$ we need to take $\alpha<d / 2$, in which case $C$ also depends on $\alpha$. For the inequality (4.6) to hold it is necessary to include the projection $P$ (see the remark following Proposition 2.1).

It now follows from Lemma 4.1 and (4.5), (4.6) that

$$
\left\|h_{m}\left(\xi^{\prime}, \eta\right)-h_{m}(\xi, \eta)\right\| \leq C\left|\xi^{\prime}-\xi\right|^{\alpha}(1-\lambda / \Lambda)^{m+1}[1+\delta(p)]^{m-1},
$$

where $p=2 d /(d+2 \alpha)$. Note here we are using the fact that (4.1) implies that a column vector of $\mathbf{b}(\cdot)$ is in $\mathcal{H}_{\mathcal{F}}^{p}\left(\mathbf{Z}^{d}\right)$ with norm less than $2 \gamma /(1+\gamma)$. The uniform Hölder continuity of the family of functions $q(\cdot, \eta), 0<\eta \leq \Lambda$, follows from (4.7) and Lemma 4.1 by taking $p$ sufficiently close to 2 so that $(1-\lambda / \Lambda)[1+\delta(p)]<1$.

The uniform Hölder continuity of the family of functions $q(\xi, \cdot), \xi \in[-\pi, \pi]^{d}$, can be obtained in a similar way by observing that

$$
\left\|P\left[T_{\xi, \eta^{\prime}}-T_{\xi, \eta}\right]\right\|_{p, 2} \leq C\left|\left(\eta^{\prime}-\eta\right) / \Lambda\right|^{\alpha / 2},
$$

where $C$ and $p$ are as in (4.6).

For $1 \leq p \leq \infty$ let $L^{p}\left(\mathbf{Z}^{d}, \mathbf{C}^{d} \otimes \mathbf{C}^{d}\right)$ be the Banach space of $d \times d$ matrix valued functions $g: \overline{\mathbf{Z}}^{d} \rightarrow \mathbf{C}^{d} \otimes \mathbf{C}^{d}$ with norm $\|g\|_{p}$ defined by

$$
\|g\|_{p}^{p}=\sup _{v \in \mathbf{C}^{d}:|v|=1} \sum_{x \in \mathbf{Z}^{d}}|g(x) v|^{p} \text { if } p<\infty, \quad\|g\|_{\infty}=\sup _{v \in \mathbf{C}^{d}:|v|=1}\left[\sup _{x \in \mathbf{Z}^{d}}|g(x) v|\right],
$$

where $|g(x) v|$ is the Euclidean norm of the vector $g(x) v \in \mathbf{C}^{d}$. We similarly define spaces $L^{p}\left([-\pi, \pi]^{d} \times \Omega, \mathbf{C}^{d} \otimes \mathbf{C}^{d}\right)$ of $d \times d$ matrix valued functions $g:[-\pi, \pi]^{d} \times \Omega \rightarrow$ $\mathbf{C}^{d} \otimes \mathbf{C}^{d}$ with norm $\|g\|_{p}$ defined by

$$
\begin{aligned}
&\|g\|_{p}^{p}=\sup _{v \in \mathbf{C}^{d}:|v|=1} \frac{1}{(2 \pi)^{d}} \int_{[-\pi, \pi]^{d}}\left\langle|g(\xi, \cdot) v|^{2}\right\rangle^{p / 2} d \xi \quad \text { if } p<\infty \\
&\|g\|_{\infty}=\sup _{v \in \mathbf{C}^{d}:|v|=1}\left[\sup _{\xi \in[-\pi, \pi]^{d}}\left\langle|g(\xi, \cdot) v|^{2}\right\rangle^{1 / 2}\right] .
\end{aligned}
$$

For $\eta>0, m=1,2, \ldots$, we define an operator $T_{m, \eta}$ from functions $g: \mathbf{Z}^{d} \rightarrow \mathbf{C}^{d} \otimes \mathbf{C}^{d}$ to periodic functions $T_{m, \eta} g:[-\pi, \pi]^{d} \times \Omega \rightarrow \mathbf{C}^{d} \otimes \mathbf{C}^{d}$ by

$$
T_{m, \eta} g(\xi, \cdot)=\sum_{x \in \mathbf{Z}^{d}} g(x) e^{-i x \cdot \xi} \tau_{x} P \mathbf{b}(\cdot)\left[P T_{\xi, \eta} \mathbf{b}(\cdot)\right]^{m-1} .
$$

We shall be interested in showing that for certain values of $p, q$ the operator $T_{m, \eta}$ is bounded from $L^{p}\left(\mathbf{Z}^{d}, \mathbf{C}^{d} \otimes \mathbf{C}^{d}\right)$ to $L^{q}\left([-\pi, \pi]^{d} \times \Omega, \mathbf{C}^{d} \otimes \mathbf{C}^{d}\right)$, uniformly in $\eta>0$. In [6] this was already shown for $p=1, q=\infty$ and $p=q=2$, with the corresponding operator norms $\left\|T_{m, \eta}\right\|_{p, q}$ satisfying the inequalities

$$
\left\|T_{m, \eta}\right\|_{1, \infty} \leq(1-\lambda / \Lambda)^{m}, \quad\left\|T_{m, \eta}\right\|_{2,2} \leq \sqrt{d}(m+1)(1-\lambda / \Lambda)^{m} .
$$

Observe now from the proof of Proposition 4.1 that for the independent random variable environment corresponding to (4.1) we can improve upon (4.12). Thus there exists $p_{0}(\Lambda / \lambda)$ with $1<p_{0}(\Lambda / \lambda)<2$ depending only on $d$ and $\Lambda / \lambda$, such that

$$
\left\|T_{m, \eta}\right\|_{p, \infty} \leq(1-\lambda / \Lambda)^{m / 2}, \quad \text { for } 1 \leq p \leq p_{0}(\Lambda / \lambda) .
$$


It now follows from (4.12), 4.13) and the Riesz convexity theorem 23 , that

$$
\begin{array}{r}
\left\|T_{m, \eta}\right\|_{p, q} \leq \sqrt{d}(m+1)(1-\lambda / \Lambda)^{m / 2}, \quad \text { for } 1 \leq p \leq 2 \\
\text { and } 1 \geq \frac{1}{p}+\frac{1}{q} \geq 1-\left[1-\frac{1}{p_{0}(\Lambda / \lambda)}\right]\left[1-\frac{2}{q}\right] .
\end{array}
$$

We can use (4.14) to obtain an improvement on Proposition 2.2 in the case $|m|=1$.

Lemma 4.2. Suppose $d \geq 2$ and $(\Omega, \mathcal{F}, P)$ in Proposition 2.2 is the Bernoulli environment corresponding to (4.1) Then in the case $|m|=1$ the derivative (2.30) is in the space $L^{p}\left([-\pi, \pi]^{d}\right)$ with $p=[d+\delta(\Lambda / \lambda)] /|m|$ and its norm is bounded by $\Lambda C(\Lambda / \lambda)$, for positive constants $\delta(\Lambda / \lambda)$ and $C(\Lambda / \lambda)$ depending only on $d$ and $\Lambda / \lambda \geq 1$.

Proof. Observe from (2.13) and (2.19) that

$$
\left(\frac{\partial}{\partial \xi_{j}}\right) h_{m}(\xi, \eta)=\sum_{k=1}^{m} \sum_{r=1}^{4}\left\langle\left[T_{m+1-k, \eta} g_{j, r}(\xi, \cdot)\right]^{*} T_{k, \eta} h_{j, r}(\xi, \cdot)\right\rangle
$$

for certain $d \times d$ matrix valued functions $g_{j, r}(x), h_{j, r}(x), x \in \mathbf{Z}^{d}$. The functions $g_{j, r}(\cdot), h_{j, r}(\cdot)$ are determined from their Fourier transforms (2.10) by the formula

$$
\sum_{r=1}^{4} \hat{g}_{j, r}(\zeta)^{*} \hat{h}_{j, r}(\zeta)=-\frac{\partial}{\partial \zeta_{j}}\left[\frac{e(-\zeta) e(-\zeta)^{*}}{\eta / \Lambda+e(-\zeta)^{*} e(-\zeta)}\right]
$$

which follows from (2.13). Evidently one can choose the $g_{j, r}(\cdot), h_{j, r}(\cdot)$ satisfying (4.16) so that they also satisfy the inequality

$$
\left\|g_{j, r}(x)\right\|+\left\|h_{j, r}(x)\right\| \leq \frac{C \exp [-\gamma|x| \sqrt{\eta / \Lambda}]}{(|x|+1)^{d-1 / 2}}, \quad x \in \mathbf{Z}^{d}, 0<\eta \leq \Lambda,
$$

for positive constants $C, \gamma$ depending only on $d \geq 1$. Hence we may estimate the RHS of (4.15) by using (4.14) for any $p>d /(d-1 / 2)$. Since we require $p \leq 2$ in (4.14) it is only possible to do this when $d \geq 2$. The result follows.

As in [6] we need to obtain norm estimates analogous to (4.14) on multilinear versions of the operator (4.11) in order to prove estimates on the derivatives (2.30) for $|m|>1$. For $\eta>0, k \geq 1$ and $m_{1}, m_{2}, \ldots, m_{k}=1,2, \ldots$, we define a multlinear operator $T_{m_{1}, m_{2}, \ldots, m_{k}, \eta}$ from a sequence $\left[g_{1}, g_{2}, \ldots, g_{k}\right]$ of $k$ functions $g_{j}: \mathbf{Z}^{d} \rightarrow \mathbf{C}^{d} \otimes$ $\mathbf{C}^{d}, j=1, \ldots, k$, to periodic functions $T_{m_{1}, m_{2}, \ldots, m_{k}, \eta}\left[g_{1}, g_{2}, \ldots, g_{k}\right]:[-\pi, \pi]^{d} \times \Omega \rightarrow$ $\mathbf{C}^{d} \otimes \mathbf{C}^{d}$ by

$$
T_{m_{1}, \ldots, m_{k}, \eta}\left[g_{1}, g_{2}, \ldots, g_{k}\right](\xi, \cdot)=\sum_{x_{1}, \ldots, x_{k} \in \mathbf{Z}^{d}} \prod_{j=1}^{k} g_{j}\left(x_{j}\right) e^{-i x_{j} \cdot \xi} \tau_{x_{j}} P \mathbf{b}(\cdot)\left[P T_{\xi, \eta} \mathbf{b}(\cdot)\right]^{m_{j}-1} .
$$

For $p$ satisfying $1 \leq p \leq \infty$ let $p^{\prime}$ be the conjugate of $p$, so $1 / p+1 / p^{\prime}=1$. In 6 ] the following generalization of (4.12) was obtained:

Lemma 4.3. Suppose $2 \leq q \leq \infty$ and $p_{1}, \ldots, p_{k}$ with $1 \leq p_{1}, \ldots, p_{k} \leq 2$ satisfy the identity

$$
\frac{1}{p_{1}^{\prime}}+\frac{1}{p_{2}^{\prime}}+\cdots+\frac{1}{p_{k}^{\prime}}=\frac{1}{q}
$$


If for $j=1, \ldots, k$, the function $g_{j} \in L^{p_{j}}\left(\mathbf{Z}^{d}, \mathbf{C}^{d} \otimes \mathbf{C}^{d}\right)$, then $T_{m_{1}, m_{2}, \ldots, m_{k}, \eta}\left[g_{1}, g_{2}, \ldots, g_{k}\right]$ is in $L^{q}\left([-\pi, \pi]^{d} \times \Omega, \mathbf{C}^{d} \otimes \mathbf{C}^{d}\right)$ and

$$
\left\|T_{m_{1}, m_{2}, \ldots, m_{k}, \eta}\left[g_{1}, g_{2}, \ldots, g_{k}\right]\right\|_{q} \leq d^{k / 2}(m+1)(1-\lambda / \Lambda)^{m} \prod_{j=1}^{k}\left\|g_{j}\right\|_{p_{j}},
$$

where $m=m_{1}+\cdots+m_{k}$.

For the independent random variable environment corresponding to (4.1) one can obtain an improvement of Lemma 4.3 analogous to the improvement (4.13) over (4.12).

Lemma 4.4. Suppose $(\Omega, \mathcal{F}, P)$ is the independent random variable environment corresponding to (4.1). Then there exists $p_{0}(\Lambda / \lambda)$ with $1<p_{0}(\Lambda / \lambda) \leq 2$ depending only on $\Lambda / \lambda$ and $d$ such that if $2 \leq q \leq \infty$ and

$$
\frac{1}{q} \leq \frac{1}{p_{1}^{\prime}}+\frac{1}{p_{2}^{\prime}}+\cdots+\frac{1}{p_{k}^{\prime}} \leq \frac{1}{q}+\left[1-\frac{1}{p_{0}(\Lambda / \lambda)}\right]\left[1-\frac{2}{q}\right]
$$

the function $T_{m_{1}, m_{2}, \ldots, m_{k}, \eta}\left[g_{1}, g_{2}, \ldots, g_{k}\right]$ is in $L^{q}\left([-\pi, \pi]^{d} \times \Omega, \mathbf{C}^{d} \otimes \mathbf{C}^{d}\right)$ and

$$
\left\|T_{m_{1}, m_{2}, \ldots, m_{k}, \eta}\left[g_{1}, g_{2}, . . g_{k}\right]\right\|_{q} \leq d^{k / 2}(m+1)(1-\lambda / \Lambda)^{m / 2} \prod_{j=1}^{k}\left\|g_{j}\right\|_{p_{j}} .
$$

Proof. We have already proved the lemma for $k=1$ so we consider the case $k=2$. Observe that (4.22) holds if $p_{2}=1$ in a similar way to the proof of (4.14). Evidently Lemma 4.3 implies that (4.22) also holds if $q=2$. Hence by an application of the Riesz convexity theorem we conclude that the result holds for the case $k=2$. To prove the result for $k=3$ we proceed similarly, using the fact that we have proved it for $k=2$, and Lemma 4.3 with $k=3$ and $q=2$.

Proof of Hypothesis 3.1. We argue just as in [6] to show that for $a=0$, Lemma 4.4 implies that the derivative (2.30) is in the space $L^{p}\left([-\pi, \pi]^{d}\right)$ with $p=d /(|m|-\alpha)$, and hence in $L_{w}^{p}\left([-\pi, \pi]^{d}\right)$. A similar argument holds for the difference (2.31). Since one can easily show that the proofs of Proposition 4.1 and Lemma 4.4 continue to hold for $|a| \leq C_{1} \sqrt{\lambda \eta / \Lambda^{2}}$, we have proven Hypothesis 3.1 for the environment corresponding to (4.1). It is shown in [5] how to extend the argument for the Bernoulli environment corresponding to (4.1) to general i.i.d. environments $\mathbf{a}\left(\tau_{x} \cdot\right), x \in \mathbf{Z}^{d}$. We have therefore proven Hypothesis 3.1 for $\mathbf{a}\left(\tau_{x} \cdot\right), x \in \mathbf{Z}^{d}$, i.i.d. such that (1.1) holds.

\section{MASsive FIELD THEORY ENVIRONMENT}

In this section we shall show that Hypothesis 3.1 holds if $(\Omega, \mathcal{F}, P)$ is given by the massive field theory environment determined by (1.13). The main tool we use to prove the theorem is the Brascamp-Lieb (BL) inequality [1. This is perhaps natural to expect since the BL inequality is needed to prove that the operators $\tau_{\mathbf{e}_{j}}, 1 \leq j \leq d$, on $\Omega$ are strong mixing, which by Proposition 2.1 implies the continuity of the function $q(\xi, \eta)$ in the region $\xi \in \mathbf{R}^{d}, \eta \geq 0$.

We recall the main features of the construction of the measure (1.13), where the function $V: \mathbf{R}^{d} \rightarrow \mathbf{R}$ is assumed to be $C^{2}$ uniformly convex with Hessian $V^{\prime \prime}(\cdot)$ satisfying the inequality $\lambda I_{d} \leq V^{\prime \prime}(\cdot) \leq \Lambda I_{d}$. Let $L$ be a positive even integer and $Q=Q_{L} \subset \mathbf{Z}^{d}$ be the integer lattice points in the cube centered at the origin with 
side of length $L$. By a periodic function $\phi: Q \rightarrow \mathbf{R}$ we mean a function $\phi$ on $Q$ with the property that $\phi(x)=\phi(y)$ for all $x, y \in Q$ such that $x-y=L \mathbf{e}_{k}$ for some $k, 1 \leq k \leq d$. Let $\Omega_{Q}$ be the space of all periodic functions $\phi: Q \rightarrow \mathbf{R}$, whence $\Omega_{Q}$ with $Q=Q_{L}$ can be identified with $\mathbf{R}^{N}$ where $N=L^{d}$. Let $\mathcal{F}_{Q}$ be the Borel algebra for $\Omega_{Q}$ which is generated by the open sets of $\mathbf{R}^{N}$. For $m>0$, we define a probability measure $P_{Q}$ on $\left(\Omega_{Q}, \mathcal{F}_{Q}\right)$ as follows:

$$
\begin{aligned}
& \langle F(\cdot)\rangle_{\Omega_{Q}} \\
& =\int_{\mathbf{R}^{N}} F(\phi(\cdot)) \exp \left[-\sum_{x \in Q}\left\{V(\nabla \phi(x))+\frac{1}{2} m^{2} \phi(x)^{2}\right\}\right] \prod_{x \in Q} d \phi(x) / \text { normalization, }
\end{aligned}
$$

where $F: \mathbf{R}^{N} \rightarrow \mathbf{R}$ is a continuous function such that $|F(z)| \leq C \exp [A|z|], z \in$ $\mathbf{R}^{N}$, for some constants $C, A$. Differentiating the probability density in (5.1) we see that for any $f \in \Omega_{Q}$

$$
\left\langle\left[\left(\nabla f(\cdot), V^{\prime}(\nabla \phi(\cdot))\right)+m^{2}(f(\cdot), \phi(\cdot))\right]\right\rangle_{\Omega_{Q}}=0,
$$

where $(\cdot, \cdot)$ denotes the Euclidean inner product on $L^{2}(Q)$. Hence by translation invariance of the measure (5.1) we conclude that $\langle(f, \phi)\rangle_{\Omega_{Q}}=0$ for all $f(\cdot)$. The BL inequality [1] applied to (5.1) and function $F(\phi(\cdot))=\exp [(f, \phi)]$ then yields the inequality

$$
\langle\exp [(f, \phi)]\rangle_{\Omega_{Q}} \leq \exp \left[\frac{1}{2}\left(f,\left\{-\lambda \Delta+m^{2}\right\}^{-1} f\right)\right] .
$$

The probability space $(\Omega, \mathcal{F}, P)$ on fields $\phi: \mathbf{Z}^{d} \rightarrow \mathbf{R}$ is obtained as the limit of the spaces $\left(\Omega_{Q}, \mathcal{F}_{Q}, P_{Q}\right)$ as $|Q| \rightarrow \infty$. In particular, one has from Lemma 2.2 of [4] the following result:

Proposition 5.1. Assume $m>0$ and let $F: \mathbf{R}^{k} \rightarrow \mathbf{R}$ be a $C^{1}$ function which satisfies the inequality

$$
|D F(z)| \leq A \exp [B|z|], \quad z \in \mathbf{R}^{k},
$$

for some constants $A, B$. Then for any $x_{1}, \ldots, x_{k} \in \mathbf{Z}^{d}$, the limit

$$
\lim _{|Q| \rightarrow \infty}\left\langle F\left(\phi\left(x_{1}\right), \phi\left(x_{2}\right), \ldots, \phi\left(x_{k}\right)\right)\right\rangle_{\Omega_{Q}}=\left\langle F\left(\phi\left(x_{1}\right), \phi\left(x_{2}\right), \ldots, \phi\left(x_{k}\right)\right)\right\rangle
$$

exists and is finite.

From (5.3) and the Helly-Bray theorem [2,8] one sees that Proposition 5.1 implies the existence of a unique Borel probability measure on $\mathbf{R}^{m}$ corresponding to the probability distribution of the variables $\left(\phi\left(x_{1}\right), \ldots, \phi\left(x_{m}\right)\right) \in \mathbf{R}^{m}$, and this measure satisfies (5.5). The Kolmogorov construction 2, 8, then implies the existence of a Borel measure on fields $\phi: \mathbf{Z}^{d} \rightarrow \mathbf{R}$ with finite dimensional distribution functions satisfying (5.5). This is the measure (1.13), which we have formally written as having a density with respect to Lebesgue measure. Note however that we do not have a proof of this fact. In particular, we do not know if the distribution measure for the one dimensional variable $\phi(x) \in \mathbf{R}$ is absolutely continuous with respect to Lebesgue measure. 
Proposition 5.2. Let $(\Omega, \mathcal{F}, P)$ be the probability space corresponding to the massive field theory with measure (1.13). Then the operators $\tau_{\mathbf{e}_{j}}, 1 \leq j \leq d$, on $\Omega$ are strong mixing.

Proof. It will be sufficient for us to show [21] that for any $m \geq 1$ and $x_{1}, \ldots, x_{m} \in \mathbf{Z}^{d}$,

$$
\begin{aligned}
\lim _{n \rightarrow \infty}\left\langlef \left(\phi\left(x_{1}+n \mathbf{e}_{1}\right), \ldots, \phi\right.\right. & \left.\left.\left(x_{m}+n \mathbf{e}_{1}\right)\right) g\left(\phi\left(x_{1}\right), \ldots, \phi\left(x_{m}\right)\right)\right\rangle \\
& =\left\langle f\left(\phi\left(x_{1}\right), \ldots, \phi\left(x_{m}\right)\right)\right\rangle\left\langle g\left(\phi\left(x_{1}\right), \ldots, \phi\left(x_{m}\right)\right)\right\rangle
\end{aligned}
$$

for all $C^{\infty}$ functions $f, g: \mathbf{R}^{m} \rightarrow \mathbf{R}$ with compact support. We shall just consider the case $m=1$ since the general case follows from this in a straightforward manner.

We define the function $h: \mathbf{Z} \rightarrow \mathbf{R}$ by

$$
h(n)=\left\langle f\left(\phi\left(n \mathbf{e}_{1}\right)\right) g(\phi(0))\right\rangle-\langle f(\phi(0))\rangle\langle g(\phi(0))\rangle, \quad n \in \mathbf{Z} .
$$

Then Proposition 5.1 implies that for any function $k: \mathbf{Z} \rightarrow \mathbf{R}$ of finite support,

$$
\lim _{|Q| \rightarrow \infty} \sum_{n \in \mathbf{Z}} k(n) h_{Q}(n)=\sum_{n \in \mathbf{Z}} k(n) h(n),
$$

where $h_{Q}(\cdot)$ is given by

$$
h_{Q}(n)=\left\langle f\left(\phi\left(n \mathbf{e}_{1}\right)\right) g(\phi(0))\right\rangle_{\Omega_{Q}}-\langle f(\phi(0))\rangle_{\Omega_{Q}}\langle g(\phi(0))\rangle_{\Omega_{Q}}, \quad n \in \mathbf{Z} .
$$

We assume that $Q=Q_{L}$ with $L$ large enough so that the support of $k(\cdot)$ is contained in the interval $[-L / 2+1, L / 2-1]$. Hence both $k(\cdot)$ and $h_{Q}(\cdot)$ are periodic functions on $I_{L}=\mathbf{Z} \cap[-L / 2, L / 2]$. We may therefore write the sum on the LHS of (5.8) in its Fourier representation. Thus the Fourier transform of a periodic function $F: I_{L} \rightarrow \mathbf{C}$ is the periodic function $\hat{F}: \hat{I}_{L} \rightarrow \mathbf{C}$ defined by

$$
\hat{F}(\zeta)=\sum_{x \in I_{L}} F(x) e^{i x \zeta}, \quad \zeta \in \hat{I}_{L}
$$

where $\hat{I}_{L}$ is the set of lattice points of $(2 \pi / L) \mathbf{Z}$ which lie in the interval $[-\pi, \pi]$. Then

$$
\sum_{n \in \mathbf{Z}} k(n) h_{Q}(n)=\frac{1}{2 \pi} \int_{\hat{I}_{L}} \hat{k}(\zeta) \overline{\hat{h}_{Q}(\zeta)} d \zeta
$$

with integration on $\hat{I}_{L}$ defined by

$$
\int_{\hat{I}_{L}}=\frac{2 \pi}{L} \sum_{\zeta \in \hat{I}_{L}} .
$$

We can estimate $\hat{h}_{Q}(\zeta)$ by using translation invariance of the measure (5.1) and the BL inequality. Thus translation invariance implies that

$$
\hat{h}_{Q}(\zeta)=\frac{1}{L}\langle a(f, \zeta, \phi(\cdot)) \overline{a(g, \zeta, \phi(\cdot))}\rangle_{\Omega_{Q}},
$$

where $a(f, \zeta, \phi(\cdot))$ is given by the formula

$$
a(f, \zeta, \phi(\cdot))=\sum_{n \in I_{L}}\left[f\left(\phi\left(n \mathbf{e}_{1}\right)\right)-\left\langle f\left(\phi\left(n \mathbf{e}_{1}\right)\right)\right\rangle_{\Omega_{Q}}\right] e^{i n \zeta} .
$$

The BL inequality then implies that

$$
\left\langle|a(f, \zeta, \phi(\cdot))|^{2}\right\rangle_{\Omega_{Q}} \leq \frac{L\|D f(\cdot)\|_{\infty}^{2}}{m^{2}}
$$


Hence there is a constant $C$ independent of $Q$ such that $\left|\hat{h}_{Q}(\zeta)\right| \leq C, \zeta \in \hat{I}_{L}$. Then applying the Schwarz inequality in (5.11) and using (5.8) we conclude that

$$
\left|\sum_{n \in \mathbf{Z}} k(n) h(n)\right| \leq C\left\{\sum_{n \in \mathbf{Z}} k(n)^{2}\right\}^{1 / 2} .
$$

It follows that $h(\cdot) \in L^{2}(\mathbf{Z})$, and consequently $\lim _{n \rightarrow \infty} h(n)=0$.

We shall show how the BL inequality can be used to improve the most elementary of the inequalities contained in $\S 2$. Thus let us consider an equation which differs from (2.4) only in that the projection operator $P$ has been omitted,

$$
\eta \Phi(\xi, \eta, \omega)+\partial_{\xi}^{*} \mathbf{a}(\omega) \partial_{\xi} \Phi(\xi, \eta, \omega)=-\partial_{\xi}^{*} \mathbf{a}(\omega), \quad \eta>0, \xi \in \mathbf{R}^{d}, \omega \in \Omega .
$$

For any $v \in \mathbf{C}^{d}$ we multiply the row vector (5.17) on the right by the column vector $v$ and by the function $\overline{\Phi(\xi, \eta, \omega) v}$ on the left. Taking the expectation we see that

$$
\left\|P \partial_{\xi} \Phi(\xi, \eta, \cdot) v\right\| \leq\left\|\partial_{\xi} \Phi(\xi, \eta, \cdot) v\right\| \leq \frac{\Lambda|v|}{\lambda},
$$

where $\|\cdot\|$ denotes the norm in $\mathcal{H}(\Omega)$. Let $g: \mathbf{Z}^{d} \rightarrow \mathbf{C}^{d} \otimes \mathbf{C}^{d}$ be in $L^{p}\left(\mathbf{Z}^{d}, \mathbf{C}^{d} \otimes \mathbf{C}^{d}\right)$ with norm given by (4.9). If $p=1$, then (5.18) implies that

$$
\left\|P \sum_{x \in \mathbf{Z}^{d}} g(x) \partial_{\xi} \Phi\left(\xi, \eta, \tau_{x} \cdot\right) v\right\| \leq\left\|\sum_{x \in \mathbf{Z}^{d}} g(x) \partial_{\xi} \Phi\left(\xi, \eta, \tau_{x} \cdot\right) v\right\| \leq \frac{\Lambda|v|}{\lambda}\|g\|_{1} .
$$

The BL inequality enables us to improve (5.19) to allow $g \in L^{p}\left(\mathbf{Z}^{d}, \mathbf{C}^{d} \otimes \mathbf{C}^{d}\right)$ for some $p>1$.

Proposition 5.3. Suppose $\mathbf{a}(\cdot)$ in (5.17) is as in the statement of Theorem 1.2. Then there exists $p_{0}(\Lambda / \lambda)$ depending only on $d$ and $\Lambda / \lambda$ and satisfying $1<p_{0}(\Lambda / \lambda)$ $<2$, such that for $g \in L^{p}\left(\mathbf{Z}^{d}, \mathbf{C}^{d} \otimes \mathbf{C}^{d}\right)$ with $1 \leq p \leq p_{0}(\Lambda / \lambda)$ and $v \in \mathbf{C}^{d}$,

$$
\left\|P \sum_{x \in \mathbf{Z}^{d}} g(x) \partial_{\xi} \Phi\left(\xi, \eta, \tau_{x} \cdot\right) v\right\| \leq \frac{\Lambda_{1} C|v|}{m \Lambda}\|g\|_{p},
$$

where $\Lambda_{1}$ is the constant in Theorem 1.2 and $C$ depends only on $d$ and $\Lambda / \lambda$.

Proof. As in Proposition 5.1 we first assume that $g(\cdot)$ has finite support in $\mathbf{Z}^{d}$. For a cube $Q$ containing the support of $g(\cdot)$ let $\Phi_{Q}(\xi, \eta, \cdot)$ be the solution to (5.17) with $\mathbf{a}(\phi)=\tilde{\mathbf{a}}(\phi(0)), \phi \in \Omega_{Q}$, so the random environment for (5.17) is $\left(\Omega_{Q}, \mathcal{F}_{Q}, P_{Q}\right)$. The BL inequality implies that

$$
\left\|P \sum_{x \in \mathbf{Z}^{d}} g(x) \partial_{\xi} \Phi_{Q}\left(\xi, \eta, \tau_{x} \cdot\right) v\right\|^{2} \leq \frac{1}{m^{2}} \sum_{z \in Q}\left\|\frac{\partial}{\partial \phi(z)} \sum_{x \in \mathbf{Z}^{d}} g(x) \partial_{\xi} \Phi_{Q}\left(\xi, \eta, \tau_{x} \cdot\right) v\right\|^{2} .
$$

Translation operators $\tau_{x}, x \in \mathbf{Z}^{d}$, act on functions $F_{Q}: \Omega_{Q} \rightarrow \mathbf{C}$ by $\tau_{x} F_{Q}(\phi(\cdot))=$ $F_{Q}\left(\tau_{x} \phi(\cdot)\right)$. We shall also need to use translation operators $T_{x}, x \in \mathbf{Z}^{d}$, which act on functions $G_{Q}: Q \times \Omega_{Q} \rightarrow \mathbf{C}$ by $T_{x} G_{Q}(z, \phi(\cdot))=G_{Q}(z+x, \phi(\cdot))$, so $T_{x}$ acts on the first variable of $G_{Q}(\cdot, \phi(\cdot))$. The operators $\tau_{x}, x \in \mathbf{Z}^{d}$, act on the second variable of $G_{Q}(\cdot, \phi(\cdot))$, and it is clear that they commute with the $T_{x}, x \in \mathbf{Z}^{d}$. For a $C^{1}$ function $F_{Q}: \Omega_{Q} \rightarrow \mathbf{C}$ let $d F_{Q}(\cdot, \phi(\cdot))$ denote its gradient so that

$$
d F_{Q}(z, \phi(\cdot))=\frac{\partial}{\partial \phi(z)} F_{Q}(\phi(\cdot)), \quad z \in Q
$$


One easily sees that

$$
d\left[\tau_{x} F_{Q}\right]=T_{-x} \tau_{x} d F_{Q}, \quad x \in \mathbf{Z}^{d},
$$

whence it follows from (2.3) that

$$
d\left[\partial_{j, \xi} \tau_{x} F_{Q}\right]=\left[e^{-i \mathbf{e}_{j} \cdot \xi} T_{-\mathbf{e}_{j}} \tau_{\mathbf{e}_{j}}-1\right] T_{-x} \tau_{x} d F_{Q}, \quad 1 \leq j \leq d, x \in \mathbf{Z}^{d} .
$$

Hence if we define a function $G_{Q}: Q \times \Omega_{Q} \rightarrow \mathbf{C}$ by

$$
G_{Q}(y, \phi(\cdot))=d F_{Q}\left(-y, \tau_{y} \phi(\cdot)\right), \quad y \in Q,
$$

then (5.24) implies that

$$
d\left[\partial_{j, \xi} \tau_{x} F_{Q}\right](z, \phi(\cdot))=\nabla_{j, \xi} G_{Q}\left(x-z, \tau_{z} \phi(\cdot)\right), \quad 1 \leq j \leq d, x, z \in \mathbf{Z}^{d},
$$

where $\nabla_{\xi}=\left(\nabla_{1, \xi}, \ldots, \nabla_{d, \xi}\right)$ and its adjoint $\nabla_{\xi}^{*}=\left(\nabla_{1, \xi}^{*}, \ldots, \nabla_{d, \xi}^{*}\right)$ are generalizations of the gradient operators (1.3),

$$
\begin{aligned}
& \nabla_{j, \xi} \phi(x)=e^{-i \mathbf{e}_{j} \cdot \xi} \phi\left(x+\mathbf{e}_{j}\right)-\phi(x), \quad 1 \leq j \leq d, x \in \mathbf{Z}^{d}, \\
& \nabla_{j, \xi}^{*} \phi(x)=e^{i \mathbf{e}_{j} \cdot \xi} \phi\left(x-\mathbf{e}_{j}\right)-\phi(x), \quad 1 \leq j \leq d, x \in \mathbf{Z}^{d},
\end{aligned}
$$

and act on the first variable of $G_{Q}(\cdot, \phi(\cdot))$. On taking $F_{Q}(\phi(\cdot))=\Phi_{Q}(\xi, \eta, \phi(\cdot)) v$ and defining $G_{Q}$ by (5.25), we conclude from (5.26) that (5.21) is the same as

$$
\left\|P \sum_{x \in \mathbf{Z}^{d}} g(x) \partial_{\xi} \Phi_{Q}\left(\xi, \eta, \tau_{x} \cdot\right) v\right\|^{2} \leq \frac{1}{m^{2}} \sum_{z \in Q}\left\|\sum_{x \in \mathbf{Z}^{d}} g(x) \nabla_{\xi} G_{Q}(x-z, \phi(\cdot))\right\|^{2} .
$$

We can find an equation for $G_{Q}(y, \phi(\cdot))$ by applying the gradient operator $d$ to (5.17). Thus from (5.24) we obtain the equation

$$
\begin{aligned}
\eta d F_{Q}(\cdot, \phi(\cdot))+D_{\xi}^{*} \tilde{\mathbf{a}}(\phi(0)) D_{\xi} & d F_{Q}(\cdot, \phi(\cdot)) \\
& =-D_{\xi}^{*}\left[\delta(\cdot) D \tilde{\mathbf{a}}(\phi(0))\left\{v+\partial_{\xi} F_{Q}(\phi(\cdot))\right\}\right],
\end{aligned}
$$

where the operators $D_{\xi}=\left(D_{1, \xi}, \ldots, D_{d, \xi}\right)$ and $D_{\xi}^{*}=\left(D_{1, \xi}^{*}, \ldots, D_{d, \xi}^{*}\right)$ are given by the formulae

$$
D_{j, \xi}=\left[e^{-i \mathbf{e}_{j} \cdot \xi} T_{-\mathbf{e}_{j}} \tau_{\mathbf{e}_{j}}-1\right], \quad D_{j, \xi}^{*}=\left[e^{i \mathbf{e}_{j} \cdot \xi} T_{\mathbf{e}_{j}} \tau_{-\mathbf{e}_{j}}-1\right], \quad 1 \leq j \leq d,
$$

and $\delta: Q \rightarrow \mathbf{R}$ is the Kronecker delta function, $\delta(0)=1, \delta(z)=0, z \neq 0$. Evidently for any $y \in \mathbf{Z}^{d}$ we can replace $\phi(\cdot)$ in (5.29) by $\tau_{y} \phi(\cdot)$. If we now evaluate (5.29) with $\tau_{y} \phi(\cdot)$ substituted for $\phi(\cdot)$ and with the first variable equal to $-y$ we obtain an equation for the function $G_{Q}(\cdot, \phi(\cdot))$ of (5.25),

$$
\begin{aligned}
& \eta G_{Q}(y, \phi(\cdot))+\nabla_{\xi}^{*} \tilde{\mathbf{a}}(\phi(y)) \nabla_{\xi} G_{Q}(y, \phi(\cdot)) \\
& \quad=-\nabla_{\xi}^{*}\left[\delta(-y) D \tilde{\mathbf{a}}(\phi(y))\left\{v+\partial_{\xi} F_{Q}\left(\tau_{y} \phi(\cdot)\right)\right\}\right], \quad y \in Q, \phi(\cdot) \in \Omega_{Q} .
\end{aligned}
$$

From (5.18) and (5.31) we immediately see that

$$
\left(\sum_{y \in Q}\left\|\nabla_{\xi} G_{Q}(y, \phi(\cdot))\right\|^{2}\right)^{1 / 2} \leq \frac{\Lambda_{1}}{\lambda}\left\|v+\partial_{\xi} F_{Q}(\phi(\cdot))\right\| \leq \frac{\Lambda_{1}}{\lambda}\left[1+\frac{\Lambda}{\lambda}\right]|v|,
$$

where $\Lambda_{1}$ is the constant of Theorem 1.2. Hence (5.28) implies the inequality

$$
\left\|P \sum_{x \in \mathbf{Z}^{d}} g(x) \partial_{\xi} \Phi_{Q}\left(\xi, \eta, \tau_{x} \cdot\right) v\right\| \leq \frac{\Lambda_{1}|v|}{\lambda m}\left[1+\frac{\Lambda}{\lambda}\right]\|g\|_{1} .
$$

The inequality (5.33) is evidently weaker than (5.19) since it involves the bound $\Lambda_{1}$ on the derivative of $\tilde{\mathbf{a}}(\cdot)$. The point is that the method applies by using Meyer's 
theorem to give bounds in terms of the $p$ norm of $g(\cdot)$ for some $p>1$. To see this first observe that one can take the limit $Q \rightarrow \mathbf{Z}^{d}$ in the inequality (5.28), whence we have that

$$
\left\|P \sum_{x \in \mathbf{Z}^{d}} g(x) \partial_{\xi} \Phi\left(\xi, \eta, \tau_{x} \cdot\right) v\right\|^{2} \leq \frac{1}{m^{2}} \sum_{z \in \mathbf{Z}^{d}}\left\|\sum_{x \in \mathbf{Z}^{d}} g(x) \nabla_{\xi} G(x-z, \phi(\cdot))\right\|^{2},
$$

where $G(\cdot, \phi(\cdot))$ is the solution to the equation

$$
\begin{aligned}
& \eta G(y, \phi(\cdot))+\nabla_{\xi}^{*} \tilde{\mathbf{a}}(\phi(y)) \nabla_{\xi} G(y, \phi(\cdot)) \\
& \quad=-\nabla_{\xi}^{*}\left[\delta(-y) D \tilde{\mathbf{a}}(\phi(y))\left\{v+\partial_{\xi} F\left(\tau_{y} \phi(\cdot)\right)\right\}\right], \quad y \in \mathbf{Z}^{d}, \phi(\cdot) \in \Omega,
\end{aligned}
$$

with $F(\phi(\cdot))=\Phi(\xi, \eta, \phi(\cdot)) v$. To see that the LHS of [5.28) converges as $Q \rightarrow \mathbf{Z}^{d}$ to the LHS of (5.34) we expand $\partial_{\xi} \Phi_{Q}(\xi, \eta, \cdot)$ in an $L^{2}$ convergent Neumann series as was done with the solution of (2.9) . Since $\eta>0$ each term in the corresponding expansion of the expectation on the LHS of (5.28) converges by Proposition 5.1 to the same term in the expansion of the LHS of (5.34). The tail of the expansion is uniformly small as $Q \rightarrow \mathbf{Z}^{d}$ from $L^{2}$ estimates. A similar argument shows that the RHS of (5.28) converges as $Q \rightarrow \mathbf{Z}^{d}$ to the RHS of (5.34).

The Neumann series for the solution to (5.35) is given in terms of an operator $T_{\xi, \eta}$ acting on functions $g: \mathbf{Z}^{d} \times \Omega \rightarrow \mathbf{C}^{d}$ which is analogous to the operator (2.7), (5.36)

$T_{\xi, \eta} g(z, \phi(\cdot))=\sum_{x \in \mathbf{Z}^{d}}\left\{\nabla \nabla^{*} G_{\eta / \Lambda}(x)\right\}^{*} \exp [-i x . \xi] g(x+z, \phi(\cdot)), \quad z \in \mathbf{Z}^{d}, \phi(\cdot) \in \Omega$.

Let $\mathbf{B}: \mathbf{Z}^{d} \times \Omega \rightarrow \mathbf{C}^{d} \otimes \mathbf{C}^{d}$ be defined by $\mathbf{B}(y, \phi(\cdot))=\tilde{\mathbf{b}}(\phi(y))$ where $\tilde{\mathbf{a}}(\cdot)=$ $\Lambda\left[I_{d}-\tilde{\mathbf{b}}(\cdot)\right]$. Equation (5.35) is then equivalent to the equation

$$
\nabla_{\xi} G(\cdot, \phi(\cdot))=T_{\xi, \eta}\left[\mathbf{B}(\cdot, \phi(\cdot)) \nabla_{\xi} G(\cdot, \phi(\cdot))\right]-T_{\xi, \eta} h(\cdot, \phi(\cdot)),
$$

with $h: \mathbf{Z}^{d} \times \Omega \rightarrow \mathbf{C}^{d}$ given by the formula

$$
h(y, \phi(\cdot))=\frac{1}{\Lambda} \delta(-y) D \tilde{\mathbf{a}}(\phi(y))\left\{I_{d}+\partial_{\xi} \Phi(\xi, \eta, \phi(\cdot))\right\} v, \quad y \in \mathbf{Z}^{d}, \phi(\cdot) \in \Omega .
$$

Now consider the Hilbert space $\mathcal{H}\left(\mathbf{Z}^{d} \times \Omega\right)$ of functions $g: \mathbf{Z}^{d} \times \Omega \rightarrow \mathbf{C}^{d}$ with norm $\|g\|_{2}$ given by

$$
\|g\|_{2}^{2}=\sum_{y \in \mathbf{Z}^{d}} \| g\left(y, \phi(\cdot) \|^{2},\right.
$$

where $\|g(y, \phi(\cdot))\|$ is the norm of $g(y, \phi(\cdot)) \in \mathcal{H}(\Omega)$. Evidently the function $h$ of (5.38) is in $\mathcal{H}\left(\mathbf{Z}^{d} \times \Omega\right)$ and

$$
\|h\|_{2} \leq \frac{\Lambda_{1}}{\Lambda}\left[1+\frac{\Lambda}{\lambda}\right]|v| .
$$

Since $\left\|T_{\xi, \eta}\right\| \leq 1$ and $\|\mathbf{B}(\cdot, \phi(\cdot))\| \leq 1-\lambda / \Lambda$, we conclude from (5.40) on summing the Neumann series for (5.37) that (5.32) holds for $Q \rightarrow \mathbf{Z}^{d}$.

We may define for any $q \geq 1$ the Banach space $L^{q}\left(\mathbf{Z}^{d} \times \Omega, \mathbf{C}^{d}\right)$ of functions $g: \mathbf{Z}^{d} \times \Omega \rightarrow \mathbf{C}^{d}$ with norm $\|\bar{g}\|_{q}$ given by

$$
\|g\|_{q}^{q}=\sum_{y \in \mathbf{Z}^{d}}\|g(y, \phi(\cdot))\|^{q} .
$$

As in Lemma 4.1 the operator $T_{\xi, \eta}$ is bounded on $L^{q}\left(\mathbf{Z}^{d} \times \Omega, \mathbf{C}^{d}\right)$ for $q>1$ with norm $\left\|T_{\xi, \eta}\right\|_{q} \leq 1+\delta(q)$, where $\lim _{q \rightarrow 2} \delta(q)=0$. Noting that $\|h\|_{q}$ is bounded by 
the RHS of (5.40) for all $q \geq 1$, we then conclude from (5.37) that there exists $q_{0}(\Lambda / \lambda)<2$ depending only on $d$ and $\Lambda / \lambda$ such that

$$
\left\|\nabla_{\xi} G(\cdot, \phi(\cdot))\right\|_{q} \leq \frac{C_{1} \Lambda_{1}|v|}{\Lambda}, \quad q_{0}(\Lambda / \lambda) \leq q \leq 2
$$

where the constant $C_{1}$ depends only on $d$ and $\Lambda / \lambda$. The result follows from (5.34), (5.42) and Young's inequality.

We now proceed to establish Hypothesis 3.1 for the massive field theory environment $(\Omega, \mathcal{F}, P)$ along the same lines followed in $\S 4$ for the i.i.d. environment.

Lemma 5.1. Let $T_{r, \eta}, r=1,2, \ldots, \eta>0$, be the operator (4.11). Then there exists $p_{0}(\Lambda / \lambda)$ with $1<p_{0}(\Lambda / \lambda)<2$ depending only on $d$ and $\Lambda / \lambda$, such that

$$
\left\|T_{r, \eta}\right\|_{p, \infty} \leq \frac{\Lambda_{1} r}{m \Lambda}(1-\lambda / \Lambda)^{r / 2} \quad \text { for } 1 \leq p \leq p_{0}(\Lambda / \lambda) .
$$

Proof. As in Proposition 5.3 it will be sufficient to assume $g: \mathbf{Z}^{d} \rightarrow \mathbf{C}^{d} \otimes \mathbf{C}^{d}$ has finite support and take the $Q \rightarrow \mathbf{Z}^{d}$ limit in the BL inequality for finite cubes $Q \subset \mathbf{Z}^{d}$. The inequality (5.43) follows immediately from BL in the case $r=1$. Thus we have for $v \in \mathbf{C}^{d}$ that the norm of $T_{1, \eta} g(\xi, \cdot) v \in \mathcal{H}(\Omega)$ is bounded as

$$
\left\|T_{1, \eta} g(\xi, \cdot) v\right\|^{2} \leq \frac{1}{m^{2}} \sum_{x \in \mathbf{Z}^{d}}\|g(x) D \tilde{\mathbf{b}}(\phi(0)) v\|^{2} \leq\left(\frac{\Lambda_{1}}{m \Lambda}\|g(\cdot)\|_{p}|v|\right)^{2}
$$

for any $p$ satisfying $1 \leq p \leq 2$.

For $r>1$ we write

$$
T_{r, \eta} g(\xi, \phi(\cdot)) v=P \sum_{x \in \mathbf{Z}^{d}} g(x) e^{-i x \cdot \xi} \tau_{x} \mathbf{b}(\cdot) \partial_{\xi} F_{r}(\phi(\cdot)), \quad \phi(\cdot) \in \Omega,
$$

where the functions $F_{r}(\phi(\cdot))$ are defined inductively by

$$
\begin{aligned}
\frac{\eta}{\Lambda} F_{r}(\phi(\cdot))+\partial_{\xi}^{*} \partial_{\xi} F_{r}(\phi(\cdot)) & =P \partial_{\xi}^{*}\left[\tilde{\mathbf{b}}(\phi(0)) \partial_{\xi} F_{r-1}(\phi(\cdot))\right], r>2, \\
\frac{\eta}{\Lambda} F_{2}(\phi(\cdot))+\partial_{\xi}^{*} \partial_{\xi} F_{2}(\phi(\cdot)) & =P \partial_{\xi}^{*}[\tilde{\mathbf{b}}(\phi(0)) v] .
\end{aligned}
$$

Similarly to (5.35) we define for $r \geq 2$ functions $G_{r}: \mathbf{Z}^{d} \times \Omega \rightarrow \mathbf{C}$ by

$$
G_{r}(y, \phi(\cdot))=d F_{r}\left(-y, \tau_{y} \phi(\cdot)\right), \quad y \in \mathbf{Z}^{d}, \phi(\cdot) \in \Omega .
$$

Then from (5.46) we see that the $G_{r}(y, \phi(\cdot))$ satisfy the equations

$$
\begin{aligned}
& \frac{\eta}{\Lambda} G_{r}(y, \phi(\cdot))+\nabla_{\xi}^{*} \nabla_{\xi} G_{r}(y, \phi(\cdot)) \\
&= P \nabla_{\xi}^{*}\left[\delta(-y) D \tilde{\mathbf{b}}(\phi(y)) \partial_{\xi} F_{r-1}\left(\tau_{y} \phi(\cdot)\right)+\tilde{\mathbf{b}}(\phi(y)) \nabla_{\xi} G_{r-1}(y, \phi(\cdot))\right], r>2, \\
& \frac{\eta}{\Lambda} G_{2}(y, \phi(\cdot))+\nabla_{\xi}^{*} \nabla_{\xi} G_{2}(y, \phi(\cdot))=P \nabla_{\xi}^{*}[\delta(-y) D \tilde{\mathbf{b}}(\phi(y)) v] .
\end{aligned}
$$

From (5.45) and BL we have that

$$
\begin{aligned}
& \left\|T_{r, \eta} g(\xi, \cdot) v\right\|^{2} \\
& \leq \frac{1}{m^{2}} \sum_{z \in \mathbf{Z}^{d}} \| g(z) e^{-i z \cdot \xi} D \tilde{\mathbf{b}}(\phi(z)) \partial_{\xi} F_{r}\left(\tau_{z} \phi(\cdot)\right) \\
& +\sum_{x \in \mathbf{Z}^{d}} g(x) e^{-i x \cdot \xi} \tilde{\mathbf{b}}(\phi(x)) \nabla_{\xi} G_{r}\left(x-z, \tau_{z} \phi(\cdot)\right) \|^{2}
\end{aligned}
$$




$$
\begin{aligned}
=\frac{1}{m^{2}} \sum_{z \in \mathbf{Z}^{d}} \| g(z) e^{-i z \cdot \xi} D \tilde{\mathbf{b}}(\phi(0)) \partial_{\xi} F_{r}(\phi(\cdot)) \\
\quad+\sum_{x \in \mathbf{Z}^{d}} g(x) e^{-i x \cdot \xi} \tilde{\mathbf{b}}(\phi(x-z)) \nabla_{\xi} G_{r}(x-z, \phi(\cdot)) \|^{2} .
\end{aligned}
$$

Just as in (5.44) we see that

$$
\frac{1}{m^{2}} \sum_{z \in \mathbf{Z}^{d}}\left\|g(z) e^{-i z \cdot \xi} D \tilde{\mathbf{b}}(\phi(0)) \partial_{\xi} F_{r}(\phi(\cdot))\right\|^{2} \leq\left\{\frac{\Lambda_{1}}{m \Lambda}\left(1-\frac{\lambda}{\Lambda}\right)^{r-1}\|g(\cdot)\|_{p}|v|\right\}^{2}
$$

for any $p$ satisfying $1 \leq p \leq 2$. The second term in the last expression in (5.49) can be bounded using an inequality similar to (5.42). It is clear from (5.48) that

$$
\left\|\nabla_{\xi} G_{r}(\cdot, \phi(\cdot))\right\|_{2} \leq \frac{\Lambda_{1}}{\Lambda}(r-1)\left(1-\frac{\lambda}{\Lambda}\right)^{r-1}|v| .
$$

Applying the Calderon-Zygmund theorem [22] to (5.48) we see that there exists $q_{0}(\Lambda / \lambda)<2$ depending only on $d$ and $\Lambda / \lambda$ such that

$$
\left\|\nabla_{\xi} G_{r}(\cdot, \phi(\cdot))\right\|_{q} \leq \frac{\Lambda_{1}}{\Lambda}(r-1)\left(1-\frac{\lambda}{\Lambda}\right)^{(r-1) / 2}|v|,
$$

provided $q_{0}(\Lambda / \lambda) \leq q \leq 2$. The result follows from (5.49), (5.50), (5.52) and Young's inequality.

Corollary 5.1. The function $q(\xi, \eta)$ of (2.5) with domain $\xi \in[-\pi, \pi]^{d}, 0<\eta \leq \Lambda$, is uniformly Hölder continuous. That is, there exist positive constants $C, \alpha$ with $0<\alpha \leq 1$ depending only on $d$ and $\Lambda / \lambda$, such that

$$
\left\|q\left(\xi^{\prime}, \eta^{\prime}\right)-q(\xi, \eta)\right\| \leq \frac{C \Lambda_{1}}{m}\left[\left|\xi^{\prime}-\xi\right|^{\alpha}+\left|\left(\eta^{\prime}-\eta\right) / \Lambda\right|^{\alpha / 2}\right]
$$

for $\xi^{\prime}, \xi \in[-\pi, \pi]^{d}$ and $0<\eta, \eta^{\prime} \leq \Lambda$.

Proof. We proceed as in the proof of Proposition 4.1. Instead of (4.6) we use the fact that

$$
P\left[T_{\xi^{\prime}, \eta}-T_{\xi, \eta}\right] \mathbf{b}(\cdot)\left[P T_{\xi, \eta} \mathbf{b}(\cdot)\right]^{j}=T_{j+1, \eta} g(\xi, \cdot)
$$

where

$$
g(x)=\left\{\nabla \nabla^{*} G_{\eta / \Lambda}(x)\right\}^{*}\left[e^{i x \cdot\left(\xi-\xi^{\prime}\right)}-1\right], \quad x \in \mathbf{Z}^{d} .
$$

Evidently for $0 \leq \alpha \leq 1$ one has that $g \in L^{p}\left(\mathbf{Z}^{d}, \mathbf{C}^{d} \otimes \mathbf{C}^{d}\right)$ for any $p>d /(d-\alpha)$ and $\|g\|_{p} \leq C_{p}\left|\xi^{\prime}-\xi\right|^{\alpha}$ for a constant $C_{p}$ depending only on $p$ and $d$. The Hölder continuity of $q(\xi, \eta)$ in $\xi$ then follows from Lemma 5.1. The Hölder continuity of $q(\xi, \eta)$ in $\eta$ can be obtained in a similar way.

To complete the proof of Hypothesis 3.1 for the massive field theory environment we need to prove a version of Lemma 4.4 and also that one can do analytic continuation in the variable $\xi \in \mathbf{R}^{d}$. The proof of this follows along the same lines as in $\S 4$. 


\section{MASSLESS FIELD THEORY ENVIRONMENT}

In this section we shall prove Hypothesis 3.1 for the massless field theory environment $(\Omega, \mathcal{F}, P)$ with measure given by the $m \rightarrow 0$ limit of the massive field theory measure (1.13). The measure is constructed by means of the following result proved in 4 :

Proposition 6.1. Let $F: \mathbf{R}^{k d} \rightarrow \mathbf{R}$ be a $C^{1}$ function which satisfies the inequality

$$
|D F(z)| \leq A \exp [B|z|], \quad z \in \mathbf{R}^{k d},
$$

for some constants $A, B$, and let $\langle\cdot\rangle_{m}$ denote the massive field theory expectation with measure (1.13). Then for any $x_{1}, \ldots, x_{k} \in \mathbf{Z}^{d}$, the limit

$$
\lim _{m \rightarrow 0}\left\langle F\left(\nabla \phi\left(x_{1}\right), \nabla \phi\left(x_{2}\right), \ldots, \nabla \phi\left(x_{k}\right)\right)\right\rangle_{m}=\left\langle F\left(\omega\left(x_{1}\right), \omega\left(x_{2}\right), \ldots, \omega\left(x_{k}\right)\right)\right\rangle
$$

exists and is finite.

As for the massive case, Proposition 6.1 defines a unique Borel probability measure on gradient fields $\omega: \mathbf{Z}^{d} \rightarrow \mathbf{R}^{d}$ by using the inequality derived from (5.3),

$$
\langle\exp [(f, \nabla \phi)]\rangle_{m} \leq \exp \left[|f|^{2} / 2 \lambda\right],
$$

for any function $f: \mathbf{Z}^{d} \rightarrow \mathbf{R}^{d}$ of finite support. This can most easily be seen by using a simple identity. For a function $G(\omega(\cdot))$ of vector fields $\omega: \mathbf{Z}^{d} \rightarrow \mathbf{R}^{d}$ we define its gradient $d_{\omega} G(\cdot, \omega(\cdot))$ similarly to $(5.22)$ by

$$
d_{\omega} G(z, \omega(\cdot))=\frac{\partial}{\partial \omega(z)} G(\omega(\cdot)), \quad z \in \mathbf{Z}^{d} .
$$

Thus $d_{\omega} G(z, \omega(\cdot)), z \in \mathbf{Z}^{d}$, is for fixed $\omega(\cdot)$ a vector field from $\mathbf{Z}^{d}$ to $\mathbf{R}^{d}$, and hence we may compute its divergence $\nabla^{*} d_{\omega} G(z, \omega(\cdot)), z \in \mathbf{Z}^{d}$. Then with $d$ defined as in (5.22) we have the identity

$$
d G(z, \nabla \phi(\cdot))=\nabla^{*} d_{\omega} G(z, \omega(\cdot)), \quad z \in \mathbf{Z}^{d} .
$$

The inequality (6.3) follows from (5.3) and (6.5) by setting $G(\omega(\cdot))=(f, \omega(\cdot))$.

Proposition 6.2. Let $(\Omega, \mathcal{F}, P)$ be the probability space corresponding to the massless field theory with measure given by the $m \rightarrow 0$ limit of the massive field theory measure (1.13). Then the operators $\tau_{\mathbf{e}_{j}}, 1 \leq j \leq d$, on $\Omega$ are strong mixing.

Proof. The proof follows the same lines as in the proof of Proposition 5.2. Thus for $C^{\infty}$ functions of compact support $f, g: \mathbf{R}^{d} \rightarrow \mathbf{R}$ let $h_{Q, m}: \mathbf{Z} \rightarrow \mathbf{R}$ be defined similarly to (5.9) by

$$
\begin{aligned}
h_{Q, m}(n)= & \left\langle f\left(\nabla \phi\left(n \mathbf{e}_{1}\right)\right) g(\nabla \phi(0))\right\rangle_{\Omega_{Q}, m} \\
& -\langle f(\nabla \phi(0))\rangle_{\Omega_{Q}, m}\langle g(\nabla \phi(0))\rangle_{\Omega_{Q}, m}, \quad n \in \mathbf{Z},
\end{aligned}
$$

where we have included the index $m$ to emphasize the dependence of the measure (5.11) on $m$. Following (5.14), let $a(f, \zeta, \omega(\cdot))$ be given by the formula

$$
a(f, \zeta, \omega(\cdot))=\sum_{n \in I_{L}}\left[f\left(\omega\left(n \mathbf{e}_{1}\right)\right)-\left\langle f\left(\omega\left(n \mathbf{e}_{1}\right)\right)\right\rangle_{\Omega_{Q}, m}\right] e^{i n \zeta} .
$$

Then it will be sufficient for us to show that

$$
\left\langle|a(f, \zeta, \nabla \phi(\cdot))|^{2}\right\rangle_{\Omega_{Q}, m} \leq \frac{L\|D f(\cdot)\|_{\infty}^{2}}{\lambda},
$$

since the RHS of (6.8) is independent of $m$. This follows from BL and (6.5). 
Proposition 6.3. Suppose $\mathbf{a}(\cdot)$ in (5.17) is as in the statement of Theorem 1.3. Then there exists $p_{0}(\Lambda / \lambda)$ depending only on $d$ and $\Lambda / \lambda$ and satisfying $1<p_{0}(\Lambda / \lambda)$ $<2$, such that for $g \in L^{p}\left(\mathbf{Z}^{d}, \mathbf{C}^{d} \otimes \mathbf{C}^{d}\right)$ with $1 \leq p \leq p_{0}(\Lambda / \lambda)$ and $v \in \mathbf{C}^{d}$,

$$
\left\|P \sum_{x \in \mathbf{Z}^{d}} g(x) \partial_{\xi} \Phi\left(\xi, \eta, \tau_{x} \cdot\right) v\right\| \leq \frac{\Lambda_{1} C|v|}{\Lambda \sqrt{\lambda}}\|g\|_{p},
$$

where $\Lambda_{1}$ is the constant in Theorem 1.3 and $C$ depends only on $d$ and $\Lambda / \lambda$.

Proof. We proceed as in the proof of Proposition 5.3. Thus from (6.5) and BL we see that

$\left\|P \sum_{x \in \mathbf{Z}^{d}} g(x) \partial_{\xi} \Phi_{Q}\left(\xi, \eta, \tau_{x} \cdot\right) v\right\|^{2} \leq \frac{1}{\lambda} \sum_{z \in \mathbf{Z}^{d}}\left\|\frac{\partial}{\partial \omega(z)} \sum_{x \in \mathbf{Z}^{d}} g(x) \partial_{\xi} \Phi_{Q}\left(\xi, \eta, \tau_{x} \omega(\cdot)\right) v\right\|^{2}$.

The remainder of the proof is exactly as in Proposition 5.3.

Proof of Hypothesis 3.1. This follows the same lines as the proof of Hypothesis 3.1 in $\S 5$.

\section{SECOND DIFFERENCE ESTIMATES}

In this section we show how the inequality (1.12) follows from Hypothesis 3.1 and the Delmotte-Deuschel argument [7. Our starting point is the representation (2.1) for the averaged Green's function $G_{\mathbf{a}, \eta}(x)$. We introduce a low momentum cutoff into the integral (2.1), then transform the remainder into configuration space and use the Hölder continuity results of [7] for the second derivatives of $G_{\mathbf{a}, \eta}(x)$. Thus let $\chi: \mathbf{R}^{d} \rightarrow \mathbf{R}$ be a $C^{\infty}$ function with compact support such that the integral of $\chi(\cdot)$ over $\mathbf{R}^{d}$ equals 1 . We write

$$
G_{\mathbf{a}, \eta}(x)=\left[G_{\mathbf{a}, \eta}(x)-\chi_{L} * G_{\mathbf{a}, \eta}(x)\right]+\chi_{L} * G_{\mathbf{a}, \eta}(x),
$$

where $\chi_{L}(x)=L^{-d} \chi(x / L), x \in \mathbf{R}^{d}$, and $*$ denotes convolution on $\mathbf{Z}^{d}$. Let $\hat{\chi}_{L}(\xi), \xi \in[-\pi, \pi]^{d}$, be the Fourier transform of $\chi_{L}$ restricted to the lattice $\mathbf{Z}^{d}$. Then for $L \geq 1$ and integers $m, n \geq 0$ there are constants $C, C_{m, n}$ such that

$$
\left|\hat{\chi}_{L}(0)-1\right| \leq C / L, \quad\left|\left(\nabla_{\xi}\right)^{m} \hat{\chi}_{L}(\xi)\right| \leq C_{m, n} L^{m} /[1+L|\xi|]^{n} \quad \xi \in[-\pi, \pi]^{d} .
$$

We assume that $R<|x|<2 R$ and choose $L=R^{1-\delta}$ for some $\delta>0$. Then from the first inequality of (7.2) and the Hölder continuity result of [7], one has the inequality

$$
\left|\nabla \nabla G_{\mathbf{a}, \eta}(x)-\nabla \nabla \chi_{L} * G_{\mathbf{a}, \eta}(x)\right| \leq \frac{C}{\Lambda(|x|+1)^{d+\alpha}} e^{-\gamma \sqrt{\eta / \Lambda}|x|},
$$

for some positive constants $\gamma$ depending only on $\Lambda / \lambda, d$ and $C, \alpha$ depending only on $\Lambda / \lambda, d, \delta$.

Next we consider the integral

$$
\frac{1}{(2 \pi)^{d}} \int_{[-\pi, \pi]^{d}} \frac{e^{-i \xi \cdot x} e_{k}(\xi) e_{j}(\xi)}{\eta+e(\xi)^{*} q(\xi, \eta) e(\xi)} \hat{\chi}_{L}(\xi) d \xi=\frac{e^{a . x}}{(2 \pi)^{d}} \int_{[-\pi, \pi]^{d}} e^{-i \xi \cdot x} f_{a}(\xi, \eta) d \xi,
$$

where for $a \in \mathbf{R}^{d}$ the function $f_{a}(\xi, \eta)$ is given by the formula

$$
f_{a}(\xi, \eta)=\frac{e_{k}(\xi+i a) e_{j}(\xi+i a)}{\eta+e(\xi-i a)^{*} q(\xi+i a, \eta) e(\xi+i a)} \hat{\chi}_{L}(\xi+i a) .
$$


Now observe from the second inequality of (17.2) that for any integer $n \geq 0$ there are positive constants $C, C_{n}$, where $C$ depends only on $d$ and $C_{n}$ on $d$ and $n$, such that

$$
\begin{aligned}
& \left|\hat{\chi}_{L}(\xi+i a)\right| \leq \frac{C_{n}}{[1+|L \xi|]^{n}}, \quad \xi \in[-\pi, \pi]^{d},|a| \leq 1 / L, \\
& \left|\hat{\chi}_{L}(\xi+i a)\right| \leq C e^{C|a| L}, \quad \xi \in[-\pi, \pi]^{d},|a| \geq 1 / L .
\end{aligned}
$$

We choose $|a|=C(\Lambda / \lambda) \sqrt{\eta / \Lambda}$ as in the proof of Theorem 3.1, where $C(\Lambda / \lambda)$ depends only on $\Lambda / \lambda$. Then if $|a| \geq 1 / L$, one has from (7.7) that

$$
\frac{e^{a . x}}{(2 \pi)^{d}} \int_{[-\pi, \pi]^{d}}\left|f_{a}(\xi, \eta)\right| d \xi \leq \frac{C}{\Lambda(|x|+1)^{d+1}} e^{-\gamma \sqrt{\eta / \Lambda}|x|}
$$

for some positive constants $\gamma$ depending only on $\Lambda / \lambda, d$ and $C$ depending only on $\Lambda / \lambda, d, \delta$. If $|a| \leq 1 / L$, we see from (7.6) that

$$
\frac{e^{a . x}}{(2 \pi)^{d}} \int_{[-\pi, \pi]^{d} \cap\left\{|\xi|>1 / R^{1-2 \delta}\right\}}\left|f_{a}(\xi, \eta)\right| d \xi \leq \frac{C}{\Lambda(|x|+1)^{d+1}} e^{-\gamma \sqrt{\eta / \Lambda}|x|},
$$

for some positive constants $\gamma$ depending only on $\Lambda / \lambda, d$ and $C$ depending only on $\Lambda / \lambda, d, \delta$.

For $a \in \mathbf{R}^{d}$ we define $g_{a}(\xi, \eta)$ similarly to $f_{a}(\xi, \eta)$ by

$$
g_{a}(\xi, \eta)=\frac{e_{k}(\xi+i a) e_{j}(\xi+i a)}{\eta+e(\xi-i a)^{*} q(0,0) e(\xi+i a)} \hat{\chi}_{L}(\xi+i a) .
$$

Then Hypothesis 3.1 implies that for $|a|<1 / L$ and $\alpha$ the Hölder constant in (3.4), (7.11)

$\frac{e^{a . x}}{(2 \pi)^{d}} \int_{[-\pi, \pi]^{d} \cap\left\{|\xi| \leq 1 / R^{1-2 \delta}\right\}}\left|f_{a}(\xi, \eta)-g_{a}(\xi, \eta)\right| d \xi \leq \frac{C}{\Lambda(|x|+1)^{(d+\alpha)(1-2 \delta)}} e^{-\gamma \sqrt{\eta / \Lambda}|x|}$,

for some positive constants $\gamma$ depending only on $\Lambda / \lambda, d$ and $C$ depending only on $\Lambda / \lambda, d, \delta$. On choosing $\delta>0$ in (7.11) to satisfy $(d+\alpha)(1-2 \delta)>d$, we conclude from (7.8), (7.9), (7.11) that

$$
\left|\nabla \nabla \chi_{L} * G_{\mathbf{a}, \eta}(x)-\nabla \nabla \chi_{L} * G_{\mathbf{a}_{\mathrm{hom}}, \eta}^{\mathrm{lattice}}(x)\right| \leq \frac{C}{\Lambda(|x|+1)^{(d+\alpha)}} e^{-\gamma \sqrt{\eta / \Lambda}|x|},
$$

for some positive constants $\gamma$ depending only on $\Lambda / \lambda, d$ and $C, \alpha$ depending only on $\Lambda / \lambda, d, \delta$. The inequality (1.12) follows from Lemma 3.1 and (7.3), (7.12) upon using the fact that

$$
\left|\nabla \nabla G_{\mathbf{a}_{\text {hom }}, \eta}^{\text {lattice }}(x)-\nabla \nabla \chi_{L} * G_{\mathbf{a}_{\text {hom }}, \eta}^{\text {lattice }}(x)\right| \leq \frac{C}{\Lambda(|x|+1)^{d+\delta}} e^{-\gamma \sqrt{\eta / \Lambda}|x|},
$$

for some positive constants $\gamma, C$ depending only on $\Lambda / \lambda, d$.

\section{RATE OF CONVERGENCE IN HOMOGENIZATION}

We turn to the proof of Theorem 1.4. The starting point for this is the Fourier representation for the solution $u(x, \eta, \omega)$ of (1.2),

$$
\begin{aligned}
& u(x, \eta, \omega)=\frac{1}{(2 \pi)^{d}} \int_{[-\pi, \pi]^{d}} \frac{\hat{h}(\xi) e^{-i \xi \cdot x}}{\eta+e(\xi)^{*} q(\xi, \eta) e(\xi)} \\
& \quad \times\left[1+\Phi\left(\xi, \eta, \tau_{x} \omega\right) e(\xi)\right] d \xi, \quad x \in \mathbf{Z}^{d}, \omega \in \Omega,
\end{aligned}
$$


where $\hat{h}(\cdot)$ is the Fourier transform (2.10) of $h: \mathbf{Z}^{d} \rightarrow \mathbf{C}$ and $\Phi(\xi, \eta, \omega)$ is the solution of (2.4). Evidently if we take the expectation of (8.1) with $\hat{h}(\cdot) \equiv 1$ we obtain (2.1).

Proof of Theorem 1.4. Taking $h(x)=\varepsilon^{2} f(\varepsilon x), x \in \mathbf{Z}^{d}$, we have from (8.1) that

$$
\begin{aligned}
u(x / \varepsilon, & \left.\varepsilon^{2} \eta, \omega\right) \\
= & \frac{1}{(2 \pi)^{d}} \int_{[-\pi / \varepsilon, \pi / \varepsilon]^{d}} \frac{\hat{f}_{\varepsilon}(\zeta) e^{-i \zeta . x}}{\eta+\varepsilon^{-2} e(\varepsilon \zeta)^{*} q\left(\varepsilon \zeta, \varepsilon^{2} \eta\right) e(\varepsilon \zeta)} \\
& \times\left[1+\Phi\left(\varepsilon \zeta, \varepsilon^{2} \eta, \tau_{x / \varepsilon} \omega\right) e(\varepsilon \zeta)\right] d \zeta, \quad x \in \varepsilon \mathbf{Z}^{d}, \omega \in \Omega,
\end{aligned}
$$

where

$$
\hat{f}_{\varepsilon}(\zeta)=\sum_{y \in \varepsilon \mathbf{Z}^{d}} \varepsilon^{d} f(y) e^{i y \cdot \zeta}, \quad \zeta \in \mathbf{R}^{d}
$$

We also have that

$$
u_{\mathrm{hom}}(x, \eta)=\frac{1}{(2 \pi)^{d}} \int_{\mathbf{R}^{d}} \frac{\hat{f}(\zeta) e^{-i \zeta . x}}{\eta+\zeta^{*} q(0,0) \zeta} d \zeta,
$$

where $\hat{f}(\cdot)$ is the Fourier transform of $f(\cdot)$,

$$
\hat{f}(\zeta)=\int_{\mathbf{R}^{d}} f(y) e^{i y \cdot \zeta} d y, \quad \zeta \in \mathbf{R}^{d} .
$$

Since $f: \mathbf{R}^{d} \rightarrow \mathbf{R}$ is $C^{\infty}$ of compact support it follows from (8.3), (8.5) that

$$
\begin{gathered}
\sup _{0<\varepsilon \leq 1, \zeta \in[-\pi / \varepsilon, \pi / \varepsilon]^{d}}\left|\hat{f}_{\varepsilon}(\zeta)\right|\left(1+|\zeta|^{2}\right)^{N}<\infty, \\
\sup _{0<\varepsilon \leq 1, \zeta \in[-\pi / \varepsilon, \pi / \varepsilon]^{d}}\left|\hat{f}_{\varepsilon}(\zeta)-\hat{f}(\zeta)\right| / \varepsilon^{2}\left[1+|\zeta|^{2}\right]<\infty,
\end{gathered}
$$

where $N$ in (8.6) can be arbitrarily large. It follows from (8.2), (8.4), (8.6) and Hypothesis 3.1, equation (3.4), that there are positive constants $C, \alpha$ such that

$$
\sup _{x \in \varepsilon \mathbf{Z}^{d}}\left|\left\langle u\left(x / \varepsilon, \varepsilon^{2} \eta, \cdot\right)\right\rangle-u_{\text {hom }}(x, \eta)\right| \leq C \varepsilon^{\alpha} .
$$

To complete the proof of the theorem we need to estimate the variance of $u\left(x / \varepsilon, \varepsilon^{2} \eta, \cdot\right)$. This was already carried out in [5] for the case of i.i.d. variables. To see this more generally we observe from (2.4) that

$$
\Phi(\xi, \eta, \omega)=P \sum_{x \in \mathbf{Z}^{d}} \nabla G_{\eta / \Lambda}(x) \exp [-i \xi . x]\left\{\mathbf{b}\left(\tau_{x} \omega\right) \partial_{\xi} \Phi\left(\xi, \eta, \tau_{x} \omega\right)+\mathbf{b}\left(\tau_{x} \omega\right)\right\} .
$$

Next we have from (4.13), Lemma 5.1 and the argument of Proposition 6.3 that for all environments in the statement of the theorem there exists $p_{0}(\Lambda / \lambda)$ depending only on $\Lambda / \lambda$ with $1<p_{0}(\Lambda / \lambda)<2$ such that

$$
\begin{gathered}
\left\|P \sum_{x \in \mathbf{Z}^{d}} g(x)\left\{\mathbf{b}\left(\tau_{x} \omega\right) \partial_{\xi} \Phi\left(\xi, \eta, \tau_{x} \cdot\right)+\mathbf{b}\left(\tau_{x} \omega\right)\right\}\right\| \leq C\|g\|_{p}, \\
g \in L^{p}\left(\mathbf{Z}^{d}, \mathbf{C}^{d}\right), 1 \leq p \leq p_{0}(\Lambda / \lambda),
\end{gathered}
$$

for some constant $C$. It follows from (8.8), (8.9) that there is a constant $C$ independent of $\varepsilon$ such that

$$
\left\|\Phi\left(\varepsilon \zeta, \varepsilon^{2} \eta, \cdot\right)\right\| \leq C \varepsilon^{d(1-1 / p)-1}, \quad \zeta \in \mathbf{R}^{d} .
$$


We conclude from (8.10) that there exist positive constants $p>1$ and $C$ independent of $\varepsilon$ such that

$$
\sup _{x \in \varepsilon \mathbf{Z}^{d}} \operatorname{var}\left[u\left(x / \varepsilon, \varepsilon^{2} \eta, \cdot\right)\right] \leq C \varepsilon^{2 d(1-1 / p)} .
$$

The result follows from (8.7), (8.11).

\section{ACKNOWLEDGEMENT}

The authors would like to thank the referee for helpful remarks. This research was partially supported by NSF under grant DMS-0553487.

\section{REFERENCES}

[1] Herm Jan Brascamp and Elliott H. Lieb, On extensions of the Brunn-Minkowski and Prékopa-Leindler theorems, including inequalities for log concave functions, and with an application to the diffusion equation, J. Functional Analysis 22 (1976), no. 4, 366-389. MR0450480 (56 \#8774)

[2] Leo Breiman, Probability, Classics in Applied Mathematics, vol. 7, Society for Industrial and Applied Mathematics (SIAM), Philadelphia, PA, 1992. Corrected reprint of the 1968 original. MR.1163370(93d:60001)

[3] Luis A. Caffarelli and Panagiotis E. Souganidis, Rates of convergence for the homogenization of fully nonlinear uniformly elliptic pde in random media, Invent. Math. 180 (2010), no. 2, 301-360, DOI 10.1007/s00222-009-0230-6. MR2609244(2011c:35041)

[4] Joseph G. Conlon, PDE with random coefficients and Euclidean field theory, J. Statist. Phys. 116 (2004), no. 1-4, 933-958, DOI 10.1023/B:JOSS.0000037204.93858.f2. MR2082201 (2005e:81132)

[5] Joseph G. Conlon and Ali Naddaf, On homogenization of elliptic equations with random coefficients, Electron. J. Probab. 5 (2000), no. 9, 58 pp. (electronic), DOI 10.1214/EJP.v565. MR1768843 (2002j:35328)

[6] Joseph G. Conlon and Ali Naddaf, Green's functions for elliptic and parabolic equations with random coefficients, New York J. Math. 6 (2000), 153-225 (electronic). MR.1781430 (2001j:35282)

[7] T. Delmotte and J.-D. Deuschel, On estimating the derivatives of symmetric diffusions in stationary random environment, with applications to $\nabla \phi$ interface model, Probab. Theory Related Fields 133 (2005), no. 3, 358-390, DOI 10.1007/s00440-005-0430-y. MR2198017 (2007a:60057)

[8] Richard Durrett, Probability: theory and examples, 2nd ed., Duxbury Press, Belmont, CA, 1996. MR 1609153 (98m:60001)

[9] T. Funaki and H. Spohn, Motion by mean curvature from the Ginzburg-Landau $\nabla \phi$ interface model, Comm. Math. Phys. 185 (1997), no. 1, 1-36, DOI 10.1007/s002200050080. MR 1463032 (98f:60206)

[10] Antoine Gloria and Felix Otto, An optimal variance estimate in stochastic homogenization of discrete elliptic equations, Ann. Probab. 39 (2011), no. 3, 779-856, DOI 10.1214/10-AOP571. MR2789576 (2012j:35018)

[11] Antoine Gloria and Felix Otto, An optimal error estimate in stochastic homogenization of discrete elliptic equations, Ann. Appl. Probab. 22 (2012), no. 1, 1-28, DOI 10.1214/10AAP745. MR2932541

[12] David Gilbarg and Neil S. Trudinger, Elliptic partial differential equations of second order, 2nd ed., Grundlehren der Mathematischen Wissenschaften [Fundamental Principles of Mathematical Sciences], vol. 224, Springer-Verlag, Berlin, 1983. MR737190 (86c:35035)

[13] Bernard Helffer and Johannes Sjöstrand, On the correlation for Kac-like models in the convex case, J. Statist. Phys. 74 (1994), no. 1-2, 349-409, DOI 10.1007/BF02186817. MR1257821 (95g:82022)

[14] S. M. Kozlov, Averaging of random structures, Dokl. Akad. Nauk SSSR 241 (1978), no. 5, 1016-1019 (Russian). MR510894 (80e:60078) 
[15] Norman G. Meyers, An $L^{p}$ estimate for the gradient of solutions of second order elliptic divergence equations, Ann. Scuola Norm. Sup. Pisa (3) 17 (1963), 189-206. MR0159110 (28 \#2328)

[16] Per-Gunnar Martinsson and Gregory J. Rodin, Asymptotic expansions of lattice Green's functions, R. Soc. Lond. Proc. Ser. A Math. Phys. Eng. Sci. 458 (2002), no. 2027, 26092622, DOI 10.1098/rspa.2002.0985. MR1942800(2003j:82019)

[17] Ali Naddaf and Thomas Spencer, On homogenization and scaling limit of some gradient perturbations of a massless free field, Comm. Math. Phys. 183 (1997), no. 1, 55-84, DOI 10.1007/BF02509796. MR1461951 (98m:81089)

[18] A. Naddaf and T. Spencer, Estimates on the variance of some homogenization problems, 1998 preprint.

[19] Hirofumi Osada and Herbert Spohn, Gibbs measures relative to Brownian motion, Ann. Probab. 27 (1999), no. 3, 1183-1207, DOI 10.1214/aop/1022677444. MR1733145 (2001f:82024)

[20] G. C. Papanicolaou and S. R. S. Varadhan, Boundary value problems with rapidly oscillating random coefficients, Random fields, Vol. I, II (Esztergom, 1979), Colloq. Math. Soc. János Bolyai, vol. 27, North-Holland, Amsterdam, 1981, pp. 835-873. MR712714 (84k:58233)

[21] William Parry, Topics in ergodic theory, Cambridge Tracts in Mathematics, vol. 75, Cambridge University Press, Cambridge, 2004. Reprint of the 1981 original. MR2140546 (2005m:37003)

[22] Elias M. Stein, Singular integrals and differentiability properties of functions, Princeton Mathematical Series, No. 30, Princeton University Press, Princeton, N.J., 1970. MR0290095 (44 \#7280)

[23] Elias M. Stein and Guido Weiss, Introduction to Fourier analysis on Euclidean spaces, Princeton University Press, Princeton, N.J., 1971. Princeton Mathematical Series, No. 32. MR0304972(46 \#4102)

[24] V. V. Yurinskiŭ, Averaging of symmetric diffusion in a random medium, Sibirsk. Mat. Zh. 27 (1986), no. 4, 167-180, 215 (Russian). MR867870 (88e:35190)

[25] V. V. Jikov, S. M. Kozlov, and O. A. Oleĭnik, Homogenization of differential operators and integral functionals, Springer-Verlag, Berlin, 1994. Translated from the Russian by G. A. Yosifian [G. A. Iosif'yan]. MR1329546 (96h:35003b)

Department of Mathematics, University of Michigan, Ann Arbor, Michigan 481091109

E-mail address: conlon@umich.edu

School of Mathematics, Institute for Advanced Study, Princeton, New Jersey 08540

E-mail address: spencer@math.ias.edu 